\title{
Turbulence structure in the upper ocean: a comparative study of observations and modeling
}

\author{
Mostafa Bakhoday Paskyabi • Ilker Fer
}

Received: 28 November 2012 / Accepted: 22 January 2014 / Published online: 18 March 2014

(C) Springer-Verlag Berlin Heidelberg 2014

\begin{abstract}
Observations of turbulent dissipation rates measured by two independent instruments are compared with numerical model runs to investigate the injection of turbulence generated by sea surface gravity waves. The nearsurface observations are made by a moored autonomous instrument, fixed at approximately $8 \mathrm{~m}$ below the sea surface. The instrument is equipped with shear probes, a highresolution pressure sensor, and an inertial motion package to measure time series of dissipation rate and nondirectional surface wave energy spectrum. A free-falling profiler is used additionally to collect vertical microstructure profiles in the upper ocean. For the model simulations, we use a one-dimensional mixed layer model based on a $k-\varepsilon$ type second moment turbulence closure, which is modified to include the effects of wave breaking and Langmuir cells. The dissipation rates obtained using the modified $k-\varepsilon$ model are elevated near the sea surface and in the upper water column, consistent with the measurements, mainly as a result of wave breaking at the surface, and energy drawn from wave field to the mean flow by Stokes drift. The agreement between observed and simulated turbulent quantities is fairly good, especially when the Stokes production is taken into account.
\end{abstract}

Responsible Editor: Martin Verlaan

This article is part of the Topical Collection on the 16th biennial workshop of the Joint Numerical Sea Modelling Group (JONSMOD) in Brest, France 21-23 May 2012

M. Bakhoday Paskyabi $(\bowtie) \cdot$ I. Fer

Geophysical Institute, University of Bergen,

Allégaten 70, 5007 Bergen, Norway

e-mail: Mostafa.Bakhoday@gfi.uib.no
Keywords Wave-current interaction · Wave-turbulence interaction - Stokes drift - Turbulence - Wave breaking . GOTM $\cdot$ Shear probe $\cdot k-\varepsilon \cdot$ Langmuir circulation . Stokes production

\section{Introduction}

The upper ocean is bounded on top by the ocean surface, a complex interface across which exchanges of heat, gases, and momentum take place. Vertical transports in this boundary are controlled by dynamical processes at the near-surface boundary layer (Thorpe 1995). The processes at the surface, and the coupling between surface gravity waves, winds, and currents in the adjacent boundary layers play a key role in the global climate system (Sullivan and McWilliams 2010). Furthermore, the near-surface boundary layer is a highly turbulent region, especially when the surface is covered by intermittent breaking waves. The breaking of surface waves enhances the production of turbulent kinetic energy (TKE), and its rate of dissipation (Gemmrich and Farmer 2004), as well as the gas transfer rates across the air-sea interface (Thorpe and Humphries 1980). Thus, a better understanding of the aforementioned processes in the upper ocean requires accurate numerical predictions and careful measurements of turbulent mixing, especially in the presence of substantial wave-induced motions.

High-quality turbulence measurements in the surface layer have been made by using fixed platforms (Agrawal et al. 1992; Terray et al. 1996), ship-based instruments (Drennan et al. 1996), surface-following floats (Soloviev and Lukas 2003; Gemmrich and Farmer 2004), ascending profilers (Anis and Moum 1995; Stips et al. 2005), and laboratory experiments (Veron and Melville 2001; Veron et al. 2008; Babanin and Haus 2009). These measurements 
show that the TKE budget at the near-surface mixed layer is influenced by surface gravity waves through wave breaking, wave-current interaction, and wave-turbulence interaction. During breaking events, air is entrained into water by bubbles and water into air by ejected droplets. Furthermore, the interaction of wind-driven surface shear with the Stokes drift of the surface waves generates Langmuir circulations that enhance the vertical mixing greatly, resulting in an elevation of the production and dissipation rate of TKE (Thorpe et al. 2003; McWilliams et al. 1997). Skyllingstad and Denbo (1995) used a large eddy simulation model to simulate the Langmuir circulation and convection in the surface mixed layer. Their simulation results were consistent with observations and confirmed that the Langmuir circulations enhance the dissipation rates and decrease the vertical gradient of temperature and velocity in the boundary layer.

Progress made by direct computational methods highlights the needs for including the dynamical structure of upper-ocean gravity wave field in the numerical models. Using the idea of breaker injection of TKE and a local production-dissipation of TKE balance, Craig and Banner (1994) and Craig (1996) imposed a surface diffusion boundary condition on the TKE equations to model the wave-enhanced turbulence in the surface zone. They suggested a constant factor to relate the surface TKE flux to the wave breaking. Terray et al. (1996) scaled three different sets of near-surface dissipation rate, $\varepsilon$, measurements under breaking waves. They used the significant wave height, $H_{s}$, for scaling depth and fitted the Craig and Banner (1994) model results to their measured $\varepsilon$ data. Mellor and Blumberg (2004) developed a boundary condition with a wave parametrization dependent on the wind stress. Sullivan et al. (2007) studied the surface gravity wave effect on the upper ocean variability by large eddy simulation using stochastic breakers and vortex forcing. Kantha and Clayson (2004) revised a turbulence closure model to include Langmuir cell effects by adding Stokes drift production term to the TKE equations. Their results showed that wave breaking influenced the mixed layer properties in the upper few meters, whereas the Langmuir cells contributed to deepening of the mixed layer.

In this study, we investigate the influence of surface gravity waves on the upper ocean turbulence variability. We compare upper ocean dissipation rate measurements with simulations using the general ocean turbulence model (GOTM) (Burchard et al. 1999) that is modified to include the wave forcing effects (Bakhoday-Paskyabi et al. 2012). Observations include (i) microstructure measurements during a 5-day cruise interrupted by a storm, (ii) background current and stratification measurements from a mooring, (iii) wave bulk parameters, and (iv) near-surface dissipation rate of TKE time series from a moored instrument at about $8 \mathrm{~m}$ from the sea surface. The modeling efforts include runs using GOTM that is modified by including wave breaking, Coriolis-Stokes forcing, Stokes production term, and waveturbulence production term. Wave forcing is obtained from the near-surface high-resolution pressure data.

This paper is organized as follows. Section 2 gives a brief description of the field measurements and the experiment site. The major components of a near-surface boundary layer model with wave effects are reviewed in Section 3. Results from numerical experiments with different wave forcing terms are presented and compared with observations in Section 4 using numerical experiments and comparisons with observations. Subsequently, a summary is given.

\section{Site and measurements}

Observations of ocean microstructure, background currents, and hydrography were made during a cruise of the Research Vessel Håkon Mosby, between 25 and 30 October 2011. The measurement site was approximately $6 \mathrm{~km}$ offshore of the Havsul-I area off the west coast of Norway (Fig. 1a), the first site in Norway with a concession for an offshore wind park. Ocean turbulence was sampled using a loosely tethered free-fall profiler MSS-90L (ISW Wassermesstechnik, Germany, MSS hereafter). Ancillary atmospheric data were logged from the ship's meteorological mast at 15-m height. Before the microstructure measurements from the vessel were initiated, a Moored Autonomous Turbulence System (MATS), a Fugro-Oceanor WaveScan (WS) buoy, and an oceanographic mooring $(\mathrm{M})$ were deployed. The relative positions of the deployed instruments are shown in Fig. $1 b$. The water depth at the measurement site is approximately $130 \mathrm{~m}$. MATS and M sampled for an extended duration, covering the cruise period reported here, and were recovered on 10 January 2012 and 6 March 2012, respectively. In this study, only the portion of the data set covering the cruise period is presented.

\subsection{Instrument details}

MSS was equipped with precision conductivity, temperature, depth (CTD) sensors, two air-foil shear probes, and one fast-response bead thermistor (Thermometrics FP07) (Fer 2006). MSS measurements were made within a 1$\mathrm{km}$ radius centered at $62^{\circ} 50^{\prime} \mathrm{N}$ and $6^{\circ} 9^{\prime} \mathrm{E}$. A total of 256 profiles were collected in 4 sets of $51,133,18$, and 54 casts, for durations of $6.9,15.7,2.0$, and $6.8 \mathrm{~h}$ (Table 1). During each set, the profiler was kept in water for five to six successive casts, recovered for repositioning the vessel, and redeployed. During the deployments, the ship drifted downwind away from the instrument, and all measurements were 100-200 m away from the vessel. The sampling concentrated in the upper 80-90 m, but occasionally deeper profiles 


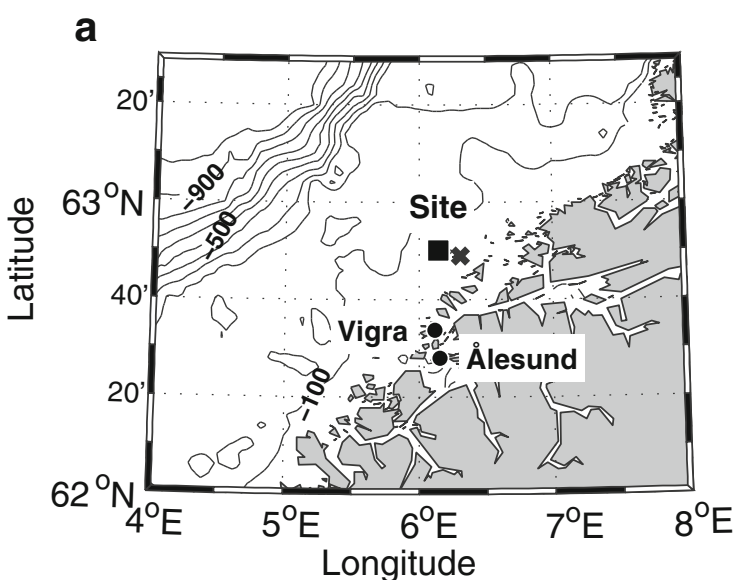

Fig. 1 a Map showing the location of the cruise site (square), Havsul-I area (cross), and the meteorological station Vigra. b Detail of the deployments during the cruise. $M$ mooring, MATS moored

(to $130-150 \mathrm{~m}$ ) were collected ( 45 out of 256 profiles). The typical sampling period between the casts was about $8 \mathrm{~min}$. The main parameter measured by MSS, reported here, is the dissipation rate of TKE, $\varepsilon$.

WS is a surface buoy equipped with a directional wave sensor, ocean surface temperature, salinity and current sensors, and a 5-m mast with standard meteorological sensors including an incoming solar radiation sensor. Due to a failure in the atmospheric mast of WS, the buoy was recovered after only 2 days of operation. The limited record from WS is used to verify the inferred wave parameters from MATS, as well as for confirming that the atmospheric data obtained from a nearby meteorological station (Vigra, Fig. 1a) are representative of the cruise site.

Background measurements collected at the mooring line at $\mathrm{M}$ included current measurements from an uplooking RD-Instruments Sentinel $300 \mathrm{kHz}$ acoustic Doppler current profiler (ADCP) located at $80 \mathrm{~m}$, and from a pair of Nortek Aquadopp current meters at 88 and $110 \mathrm{~m}$; and temperature and salinity measurements from Sea-Bird Electronics (SBE) loggers (six MicroCats and two SeaCats) distributed evenly in the vertical. The ADCP sampled the horizontal currents in 2-m vertical bins. Sampling interval was 2 min for

Table 1 MSS profiling periods during the experiment in October 2011

\begin{tabular}{lllcr}
\hline & Start & End & Duration & Casts \\
\hline Set 1 & $2514: 56$ & $2521: 49$ & 6.9 & 51 \\
Set 2 & $2706: 05$ & $2721: 49$ & 15.7 & 133 \\
Set 3 & $2806: 04$ & $2808: 04$ & 2 & 18 \\
Set 4 & $2917: 03$ & $2923: 53$ & 6.8 & 54 \\
\hline
\end{tabular}

Start and end times are given as day in October and time in UTC. The duration is in hours

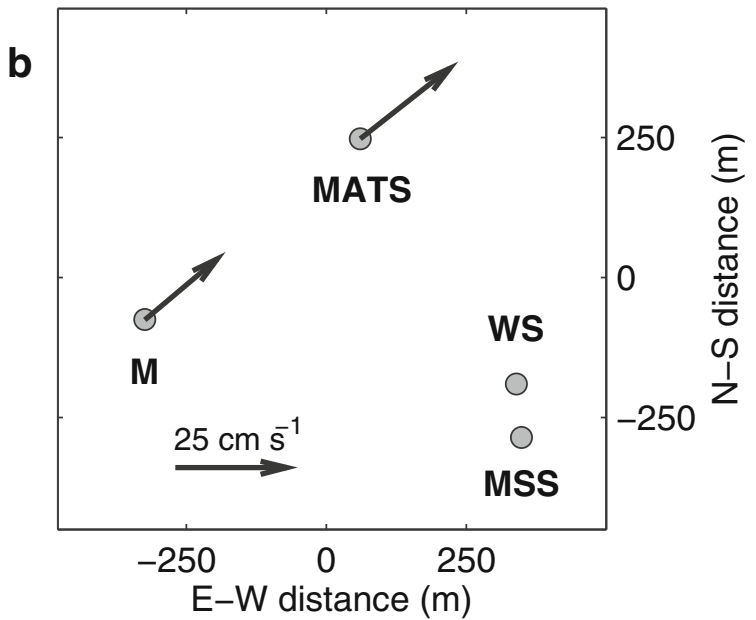

autonomous turbulence system, WS WaveScan buoy, and MSS microstructure profiler. Depth averaged currents measured by the ADCPs at $\mathrm{M}$ and MATS are also shown (arrows) with scale indicated

MicroCats, $1 \mathrm{~h}$ for SeaCats, $15 \mathrm{~min}$ for Aquadopps, and $5 \mathrm{~min}$ for the ADCP. M was deployed at the 128-m isobath.

Near-surface turbulence measurements were made using MATS, an instrument designed to collect microstructure time series at a fixed level for an extended period (Fer and Bakhoday-Paskyabi 2014). MATS consists of a lowdrag buoy as the main platform fitted with a modified micro-Rider turbulence package (Rockland Scientific International, Canada) and a $6 \mathrm{MHz}$ Nortek Vector acoustic Doppler velocimeter. The buoy has sufficient buoyancy to be used as the upper element in a mooring line. A swivel allows the instrument to align with the current, pointing the sensors toward the undisturbed free flow. The mooring line of MATS was equipped with additional oceanographic sensors to sample background currents and hydrography (2 MicroCats at 10 and $30 \mathrm{~m}$; 4 SBE39 temperature loggers at $15,20,40$, and $50 \mathrm{~m}$; an uplooking RDI Sentinel $300 \mathrm{kHz}$ ADCP at $60 \mathrm{~m}$, all sampling at intervals identical to those at M). Data were recovered from all instruments but the uppermost MicroCat.

The sensors on MATS included two air-foil shear probes, two FP07 thermistors, a pressure transducer, a dual axis vibration sensor, a dual axis inclinometer, a six-axis motion sensor (measuring angular rates and accelerations), and a magnetometer. All turbulence sensors of the microRider and the sensor head of the Vector protruded horizontally, about $25 \mathrm{~cm}$ from the nose of the buoy, pointing to the mean flow. No probe guard was installed. MATS was located at about 8$\mathrm{m}$ depth from the surface. The mean and standard deviation of the pressure record for the duration of the deployment was $8.2 \pm 0.7 \mathrm{dbar}$. MATS sampled 15 -min bursts every $1 \mathrm{~h}$, at $512 \mathrm{~Hz}$ for the turbulence channels, at $64 \mathrm{~Hz}$ for the slow channels including the motion sensors, and at $16 \mathrm{~Hz}$ for the Vector. 
For details of the instrument and data processing, the reader is referred to Fer and Bakhoday-Paskyabi (2014) and Bakhoday-Paskyabi and Fer (2013). In this study, dissipation rate measurements from the shear probes are used. MATS is not designed to move with the wave motions; the use of velocimeter data for turbulence measurements would require substantial corrections for the platform motion. We use the ADV data to infer the wave-band rms velocities, and the mean flow past sensors. The dissipation rate of TKE is measured by the shear probes, in the dissipation range of the turbulence spectrum, in the frequency range unaffected by wave motion (see Section 4.4)

\subsection{Dissipation rate calculations}

Time series of dissipation rate is calculated using data from the shear probes of MATS. The output from the analog differentiator of the shear probe is converted to shear using the known sensitivity of the probe and water velocity past the sensor, recorded by the Vector. Two-second smoothed velocity sampled at $16 \mathrm{~Hz}$ is interpolated to the turbulence channel sampling rate before obtaining the shear using the Taylor's hypothesis. Shear probes are mounted orthogonal to each other, and measure the components $\partial w / \partial x$ and $\partial v / \partial x$. The 15 -min burst time series are analyzed in halfoverlapping 60-s segments. The frequency band 1 to $20 \mathrm{~Hz}$ of the shear spectrum, not affected by the wave motion, is used to ensure that the dissipation rates are not contaminated by the platform motion (Section 4.4). The frequency spectra of shear, $\Psi(f)$, are converted to the wavenumber spectra using

$\Psi(\tilde{k})=\mathcal{U} \Psi(f)$, and $\tilde{k}=f / \mathcal{U}$,

where $\mathcal{U}$ is the mean flow past the sensor, averaged over the 60 -s duration of the segment, $\tilde{k}$ is the wavenumber in cycles per meter (cpm). Each shear spectrum is corrected for the probe's spatial response. Vibration contamination is removed using the accelerometer data, using the method outlined in Goodman et al. (2006). The dissipation rate of TKE for each segment is calculated using the isotropic relation for one shear component, for example for $\partial w / \partial x$, as

$\varepsilon=\frac{15}{2} v \overline{\left(\frac{\partial w}{\partial x}\right)^{2}}=\frac{15}{2} v \int_{2}^{k_{c}} \Psi(\tilde{k}) d \tilde{k}$,

where $v$ is the kinematic viscosity, and overbar denotes the spatial or time average. Integration is carried out between $2 \mathrm{cpm}$ and an upper cutoff wavenumber $k_{c}$, using an iterative procedure (Fer and Bakhoday-Paskyabi 2014), where $k_{c}$ is always less than $40 \mathrm{cpm}$ to ensure a noise-free spectrum. The remaining part of the spectrum (the variance which is not accounted for) is integrated using the empirical Nasmyth spectrum (Oakey 1982). Ideally, the dissipation measurements from the two shear probes would be averaged; however, the shape of shear spectra from probe 2 and inferred dissipation rates are found to be erroneous, and we use only the measurements from probe 1 . Then, the mean dissipation rate at each 15-min burst is obtained using the maximum likelihood estimator technique (Section 4.5).

The processing of MSS data is similar, and follows Fer (2006). In obtaining $\varepsilon$ profiles from MSS, fall speed inferred from the rate of change of pressure, approximately $0.6 \mathrm{~m} \mathrm{~s}^{-1}$, is used in Taylor's hypothesis. One-second $(\approx$ $0.6 \mathrm{~m}$ ) long segments are analyzed, and 1-m vertically averaged $\varepsilon$ profiles are obtained by averaging over both shear probes.

\section{Near-surface boundary layer model with wave effects}

In this section, we briefly review the model of upper ocean mixed layer. Model equations are considered in Cartesian $(z, t)$ coordinates where $z$ points positive upward and all quantities are dimensional. The momentum balance equations are written in terms of Coriolis-Stokes forcing and vortex force to account the effects of Langmuir circulation. To meet the one-dimensionality assumption, we assume horizontal homogeneity of the velocity and temperature fields, and ignore the advection terms in the remaining of the paper (except for the vortex force term in Eq. 3 to highlight the general form of wave-current interaction that is ignored in the one-dimension model).

\subsection{Wave energy balance equation}

A realistic representation of turbulence variability in the upper ocean requires reliable estimates of energy and momentum fluxes to the water column. Numerical modeling of surface gravity waves offers a way to estimate these fluxes by solving the energy balance equation. For deep water,

$\frac{\partial S}{\partial t}+\mathbf{c}_{g} \nabla S=S_{i n}+S_{d i s s}+S_{n l}$

where $S=S(f, \theta)$ is the two-dimensional wave energy spectrum which gives the energy distribution of the ocean waves over frequency, $f$, and propagation direction, $\theta$. In Eq. $2, \mathbf{c}_{g}$ is the group velocity, and $S_{i n}, S_{d i s s}$, and $S_{n l}$ describe the generation of ocean waves by wind, the dissipation of waves, and the nonlinear wave energy transfer, respectively (Komen 1987). Details of the source function relations used in this study can be found in Janssen (1991) and Bakhoday-Paskyabi et al. (2012). 


\subsection{Coriolis-Stokes forcing and vortex force}

In a rotary frame of reference, the interaction between the Stokes drift and the planetary vorticity yields a force on the Eulerian momentum, which is referred to as Coriolis-Stokes forcing (CSF). Hasselmann (1970) illustrated that in the presence of the Earth's rotation, the horizontal and vertical wave orbital velocities are no longer in quadrature, but create a new wave-induced forcing to the water column. This body force exerted by waves can be presented as the divergence of a wave-induced stress, $\rho \overline{\tilde{v}} \tilde{w}$, where $\tilde{v}$ and $\tilde{w}$ are the components of the rapidly varying wave orbital velocities along the wave crest and vertical directions, respectively, and $\rho$ is water density (Hasselmann 1970; Polton et al. 2005; Rascle and Ardhuin 2009; Bakhoday-Paskyabi et al. 2012).

In the non-steady case, the (quasi) Eulerian mean currents (wave-phase-averaged mean flow) are governed by the wave-modified momentum equations as follows (McWilliams et al. 1997):

$$
\begin{aligned}
\frac{\partial \mathbf{u}}{\partial t} & =-f_{\text {cor } \hat{z} \times \mathbf{u}}+\mathbf{F}_{C S F}-\frac{\partial}{\partial z} \underbrace{\overline{\mathbf{u}^{\prime} w^{\prime}}}_{1}-\frac{1}{\rho_{0}} \nabla p^{*} \\
& +\underbrace{\mathbf{u}_{s} \times(\nabla \times \mathbf{u})}_{2}+\mathbf{F}_{d s}(z),
\end{aligned}
$$

where the boldface denotes vector quantities, $\mathbf{u}=(u, v, w)$ is the quasi-Eulerian mean current that can be understood as the Eulerian mean current (Jenkins 1987), $\mathbf{u}^{\prime}=\left(u^{\prime}, v^{\prime}, w^{\prime}\right)$ is the random turbulent velocity fluctuation, overbar denotes time averaging, $\mathbf{u}_{s}=\left(u_{s}, v_{s}, 0\right)$ is the Stokes drift, $f_{c o r}$ is the Coriolis parameter, and $\hat{z}$ denotes a vertical unit vector pointing positive upward. The CSF term is defined by Hasselmann (1970) as

$\mathbf{F}_{C S F}=-f_{\text {cor }} \times \mathbf{u}_{S}$.

The unknown Reynolds stresses $\overline{u^{\prime} w^{\prime}}$ and $\overline{v^{\prime} w^{\prime}}$ in term 1 are defined using the well-known closure relations as follows:

$\overline{u^{\prime} w^{\prime}}=-v_{t} \frac{\partial \bar{u}}{\partial z}$, and $\overline{v^{\prime} w^{\prime}}=-v_{t} \frac{\partial \bar{v}}{\partial z}$,

where $v_{t}$ is the vertical eddy viscosity. Term 2 is the vortex force (McWilliams et al. 1997; Tang et al. 2007) which is responsible for producing Langmuir circulation. $p^{*}$ is the modified pressure by the Stokes drift (Bernoulli term):

$p^{*}=p_{a}+\rho_{0} g \xi+g \int \rho d z+\frac{1}{2}\left(\left|\mathbf{u}+\mathbf{u}_{s}\right|^{2}-|\mathbf{u}|^{2}\right)$,

where $p_{a}$ is the air pressure, $\rho_{0}$ is the reference density, $g$ is the gravitational acceleration, $\xi$ is the vertical displacement of the free surface, and $|\cdot|$ denotes magnitude of a vector. The last term in Eq. 3 denotes the wave-induced transfer of momentum from wave to the mean current as a result of wave energy dissipation, that is defined as follows (Jenkins 1987):

$\mathbf{F}_{d s}(z)=-4 \pi \int_{f} \int_{\theta} f S_{d i s s}(f, \theta) \mathbf{k} \exp (-2 k|z|) d \theta d f$,

where $\mathbf{k}=k(\cos \theta, \sin \theta)$ is the wavenumber vector that can be prescribed through the dispersion relation (36). Here, the wave-induced momentum transfer has an exponential decay away from the sea surface by a vertical distribution of $\exp (-2 k|z|)$.

\subsection{Coupling through wave-induced stress}

Surface gravity waves influence the air flow (Miles 1957). Interaction between wave-induced oscillations and the mean air flow in the turbulent atmospheric boundary layer induces pressure fluctuations on the water surface, in phase with the surface slope, which cause transfer of energy and momentum from the mean air flow to the wave field (Janssen 1989; Jenkins 1992). The dependence of the air-sea momentum flux on the wave field may be approximated by a wave-dependent air-side aerodynamic roughness length, $z_{0}^{a}$, which increases with wind speed. Changes in $z_{0}^{a}$ feedback into the wind speed profile $U(z)$ (under neutral conditions) via the logarithmic turbulent boundary layer relation as follows:

$U(z)=\left(u_{*}^{a} / \kappa\right) \log \left(z / z_{0}^{a}\right)$,

where $\kappa \approx 0.4$ is the von Kármán constant. In this study, the air-side surface roughness length is computed following Janssen (1989), using a variable Charnock parameter $\alpha_{v}$ :

$z_{0}^{a}=\left(\alpha_{v}\left(u_{*}^{a}\right)^{2} / g\right)+z_{0 \min }$,

where $u_{*}^{a}=\sqrt{\left|\tau_{\text {wind }}\right| / \rho_{a}}$ is the air-side friction velocity in $\mathrm{m} \mathrm{s}^{-1}, \tau_{\text {wind }}$ is the wind stress (air-sea momentum flux) in $\mathrm{N} \mathrm{m}^{-2}, \rho_{a}$ is the air density, $z_{0 \text { min }}=1.59 \times 10^{-5} \mathrm{~m}$ is the minimum roughness length, and

$\alpha_{v}=\min \left(\frac{\alpha_{\min }}{\sqrt{1-R}}, \alpha_{\max }\right)$,

where $R=\left|\tau_{\text {wave }}\right| /\left|\tau_{\text {wind }}\right|$. The variable Charnock parameter $\alpha_{v}$ has minimum and maximum values of $\alpha_{\min }=0.01$ and $\alpha_{\max }=0.31$, respectively. When no wave information is available, a constant $\alpha_{v}=0.0185$ is used. $\tau_{\text {wave }}$ is the wave-induced stress used in the surface momentum flux boundary condition to impose a reduction in wind stress as a result of generation of surface gravity waves:

$v_{t} \frac{\partial \mathbf{u}}{\partial z}=\frac{1}{\rho_{0}}\left[\tau_{\text {wind }}-\tau_{\text {wave }}\right]$.

Following Jenkins (1987), we define

$\tau_{\text {wave }}=\rho_{0} \int_{0}^{2 \pi} \int_{0}^{\infty} \omega \hat{\mathbf{k}} S_{i n}(f, \theta) d f d \theta$, 
where $S_{i n}$ is the wind energy input source term (23), $\omega=$ $2 \pi f$ is angular frequency, and $\mathbf{k}=k \hat{\mathbf{k}}$ is the horizontal wavenumber with modulus $k$ and direction $\hat{\mathbf{k}}$.

\subsection{Langmuir circulation and turbulence closure}

The Stokes drift and the associated vortex force have been considered as the responsible mechanisms for driving Langmuir circulation (LC), as a result of the interaction with the wind-driven surface shear (Kantha and Clayson 2004). Furthermore, the Stokes drift production enhances vertical mixing by fluxing energy through the sea surface, and by TKE production associated with Stoke drift shear (Anis and Moum 1995; Gerbi et al. 2009). To include the Stokes production term in the TKE budget equation, we use second moment turbulence closure models that are derived from the Reynolds averaged Navier-Stokes (RANS) equations. They are then closed by assuming a local equilibrium and finding second moment parameterizations for the unknown third moments.

\subsubsection{The $k$ model}

As a consequence of adding the CSF and the vortex force to the momentum equations, the equation governing TKE per unit mass, $k$, must be modified (Here, $k$ should not be mixed with the wavenumber). Thus, the modified $k$-equation is given as follows:

$$
\begin{aligned}
\overbrace{\frac{\partial k}{\partial t}}^{1} & =\overbrace{\frac{\tau}{\rho_{0}} \cdot \frac{\partial \mathbf{u}}{\partial z}}^{2}+\overbrace{\frac{\tau}{\rho_{0}} \cdot \frac{\partial \mathbf{u}_{s}}{\partial z}}^{3}+\overbrace{b^{\prime} w^{\prime}}^{4}-\varepsilon \\
& +\overbrace{\frac{\partial}{\partial z}\left[\frac{v_{t}}{\sigma_{k}} \frac{\partial k}{\partial z}\right]}^{5}-\overbrace{\frac{\partial T}{\partial z}}^{6},
\end{aligned}
$$

where "." denotes dot-product in the vector space, term 1 is the rate of change of TKE, term 2 is the production of TKE by mean shear $\left(P_{\text {curr }}\right)$, term 3 is the Stokes production $\left(P_{\text {Stokes }}\right)$, term 4 is the buoyancy production/dampening $\left(P_{b}\right)$, where $b^{\prime}$ is the buoyancy perturbation, $\tau=-\rho_{0}\left(\overline{u^{\prime} w^{\prime}}, \overline{v^{\prime} w^{\prime}}, 0\right)$ is the Reynolds stress vector, and $\varepsilon$ is the dissipation rate of TKE per unit mass. Term 5 represents the vertical diffusive transport of TKE, where $\sigma_{k}$ denotes the Schmidt number for TKE. The delicate coupling between turbulence and pressure fluctuations near the sea surface is given in term 6 , where $T=\overline{p^{\prime} w^{\prime}} / \rho_{0}+$ $\overline{\mathbf{u}^{\prime} \mathbf{u}^{\prime} w^{\prime}}$ is the vertical transport of TKE. However, due to difficulty in estimating the pressure term, we determine it from the energy transport caused by wave energy dissipation into the interior ocean (Janssen 2012). By ignoring the triple-correlation term from $T$ and assuming that wave breaking/white capping generates the pressure work near the sea surface, term 6 can be approximated from the vertical derivative of

$T(z)=g \iint S_{\text {diss }}(f, \theta) \exp \left(-|z| / z_{0}\right) d f d \theta$,

where $z_{0}$ is the water-side roughness length (Section 3.6).

\subsubsection{The \& model}

The dissipation rate of TKE can be transported via

$$
\begin{aligned}
\frac{\partial \varepsilon}{\partial t} & =C_{1} \frac{\varepsilon}{k}\left[\frac{\vec{\tau}}{\rho_{0}} \cdot \frac{\partial \mathbf{u}}{\partial z}+C_{4} \frac{\vec{\tau}}{\rho_{0}} \cdot \frac{\partial \mathbf{u}_{s}}{\partial z}-C_{5} \frac{\partial T}{\partial z}+C_{3} \overline{b^{\prime} w^{\prime}}\right] \\
& -C_{2} \frac{\varepsilon^{2}}{k}+\frac{\partial}{\partial z}\left[\frac{v_{t}}{\sigma_{\varepsilon}} \frac{\partial \varepsilon}{\partial z}\right]
\end{aligned}
$$

where $\sigma_{\varepsilon}$ is the dissipation Schmidt number, and $C_{1}, C_{2}$, $C_{3}, C_{4}$, and $C_{5}$ are empirical coefficients. In contrast to the exact expression of the $k$ model, the $\varepsilon$ model is an empirical equation dependent on empirical constants (Burchard and Bolding 2001). $\sigma_{\varepsilon}$ is one such empirical parameter with values ranging from 0.8 to 1.11 , and for the law-of-thewall (LOW), in the case of steady state and constant stress conditions,

$\sigma_{\varepsilon, L O W}=\frac{\kappa^{2}}{\sqrt{c_{\mu}}\left(C_{2}-C_{1}\right)}$,

where $c_{\mu}$ is the stability function that is used to determine the magnitude of eddy viscosity, $v_{t}$, in the $k-\varepsilon$ model (19). Here, following Burchard (2001) and using the terminology of Stips et al. (2005), $\sigma_{\varepsilon}$ in the wave-affected boundary layer (WABL), where the shear production is relatively small compared to the dissipation, is calculated as follows:

$\sigma_{\varepsilon}=R_{1} \sigma_{\varepsilon, \mathrm{WABL}}+R_{2} \sigma_{\varepsilon, \text { LOW }}$

Here,

$R_{1}=\max \left\{0,1-\frac{P_{\text {curr }}+P_{\text {Stokes }}+P_{b}}{\varepsilon}\right\}$,
$R_{2}=\min \left\{1, \frac{P_{\text {curr }}+P_{\text {Stokes }}+P_{b}}{\varepsilon}\right\}$,

where $P_{\text {curr }}, P_{\text {Stokes }}$, and $P_{b}$ are the shear production, Stokes production, and buoyancy production/dampening terms of Eq. 12, that can be written using the eddy viscosity, $v_{t}$, and eddy diffusivity, $v_{t}^{\prime}$, as

$P_{\text {curr }}=v_{t} M^{2}, P_{\text {Stokes }}=v_{t} M_{s}, P_{b}=-v_{t}^{\prime} N^{2}$.

Here, the shear, $M$, buoyancy frequency, $N$, and wavecurrent interaction, $M_{s}$, terms are defined as follows:

$M^{2}=\frac{\partial \mathbf{u}}{\partial z} \cdot \frac{\partial \mathbf{u}}{\partial z}, M_{s}=\frac{\partial \mathbf{u}}{\partial z} \cdot \frac{\partial \mathbf{u}_{s}}{\partial z}, N^{2}=-g \frac{1}{\rho_{0}} \frac{\partial \rho}{\partial z}$. 
Using stability functions for momentum and tracers, $c_{\mu}$ and $c_{\mu}^{\prime}$, respectively, the eddy viscosity and diffusivity are obtained as follows:

$v_{t}=c_{\mu} \frac{k^{2}}{\varepsilon}$, and $v_{t}^{\prime}=c_{\mu}^{\prime} \frac{k^{2}}{\varepsilon}$.

Empirical constants for the $\varepsilon$ model used in this paper are given in Table 2, and values for $C_{3}$ are obtained depending on the stability function (Burchard and Bolding 2001; Umlauf et al. 2003; Kantha and Clayson 2004; Umlauf and Burchard 2003).

The turbulence length scale, $l$, in terms of $k$ and $\varepsilon$ is calculated as follows:

$l=\left(c_{\mu}^{0}\right)^{3} \frac{k^{3 / 2}}{\varepsilon}$,

where $c_{\mu}^{0}$ is an empirical constant (Table 2).

3.5 Wave breaking and boundary conditions

\subsubsection{Wave breaking}

Surface gravity waves enhance production of TKE and its dissipation, especially in windy conditions, producing a large deviation from the usual balance between the production, dissipation, and buoyancy terms of the TKE budget. Kitaigorodskii et al. (1983) found that turbulence level depends on surface waves, and dissipation rates measured near the sea surface are 1 or 2 orders of magnitude larger than those predicted by the LOW:

$\varepsilon_{L O W}=\frac{\left(u_{*}^{w}\right)^{3}}{\kappa|z|}$.

Gargett (1989) suggested that near the sea surface, $\varepsilon$ follows a $|z|^{-4}$ power law. Craig and Banner (1994) obtained $|z|^{-3.4}$. Terray et al. (1996) found $|z|^{-2}$ that was supported by measurements of Drennan et al. (1996). The considerable disagreement between different authors suggests that the decay of dissipation rate does not follow a simple power law, and merits further investigations.

Table 2 Empirical constants for the $k-\varepsilon$ equations following (Stips et al. 2005). Since, we will not use the wave breaking production term, the empirical coefficient $C_{5}$ is excluded from table

\begin{tabular}{ll}
\hline Parameter & Value \\
\hline$C_{1}$ & 1.44 \\
$C_{2}$ & 1.92 \\
$C_{4}$ & 0.8 \\
$\sigma_{k}$ & 1 \\
$\sigma_{\varepsilon, \text { LOW }}$ & 1.2 \\
$\sigma_{\varepsilon, \mathrm{WABL}}$ & 2.01 \\
$\kappa$ & 0.41 \\
$c_{\mu}^{0}$ & 0.55 \\
\hline
\end{tabular}

A number of one-dimensional vertical models have been developed to capture the effects of surface gravity waves. Craig and Banner (1994) and Craig (1996) employed the level $2 \frac{1}{2}$ turbulence closure model of Mellor and Yamada (1982) to predict near-surface turbulence. They modeled the wave breaking effects by prescribing an additional energy injection mechanism as the surface boundary conditions to the TKE and mixing length equations. Using a 1D mixed layer model, Kantha and Clayson (2004) modified the two-equation second moment closure model of turbulence to account for the injection of TKE into the upper ocean by wave breaking and Langmuir cells. Their results showed that wave breaking enhances turbulence mixing in the upper few meters, whereas the energy input to the turbulence from the Langmuir cells drives deeper mixing. Rascle et al. (2006) used a single evolution TKE equation to study mixing due to breaking wave effects and wave-turbulence interactions based on a generalized Lagrangian mean technique. Recently, Bakhoday-Paskyabi et al. (2012) modified the GOTM system based on a theory of wave-current interaction (Jenkins 1987) by including Coriolis-Stokes forcing, wave-induced momentum reduction, and a wave-induced vertical momentum redistribution term. They emphasized the role of Stokes drift in modifying upper ocean variability among other wave forcing parameters. A common technique to simulate wave breaking in all of these 1D models is the parametrization of the TKE flux at the surface as follows:

$F_{k}=-\beta\left(u_{*}^{w}\right)^{3}$,

where $\beta$ is a parameter dependent on the wave age, the phase speed of waves, and the air-side friction velocity. Wang and Huang (2004) assumed that the surface TKE flux is equal to the wind energy input, and obtained $\beta=80$ from observations. The rate of wind energy input, $F_{k}$, to the waves is determined by integration of the wind energy input source term for all frequency and directions (Kantha and Clayson 2004; Bakhoday-Paskyabi et al. 2012):

$F_{k}=-\int_{f} \int_{\theta} \beta_{w} \frac{\rho_{a}}{\rho_{w}}\left(\frac{u_{*}^{a}}{c_{\text {phase }}}\right)^{2} \Theta^{2} \omega S(f, \theta) d \theta d f$,

where $\Theta=\max \left[0, \cos \left(\theta-\theta_{w}\right)\right], \theta_{w}$ is the mean wind direction, and $\beta_{w}$ is the Miles constant (Janssen 1991). The least squares regression between Eqs. 23 and 22 gives a proper value for $\beta$, specifically for the developed seas. For young waves with wave age roughly less than $13, \beta$ cannot be assumed constant, and the flux of TKE can be determined in terms of the wave energy dissipation as follows:

$F_{k}=g \int_{f} \int_{\theta} S_{d i s s}(f, \theta) d \theta d f$. 


\subsubsection{Boundary conditions for the $k$-equation}

The balance of vertical energy flux and TKE from breaking waves in the surface is expressed by either flux Neumann or Dirichlet boundary conditions. The flux boundary condition can be written, for example, in the $k-\varepsilon$ model as follows:

$\frac{v_{t}}{\sigma_{k}} \frac{\partial k}{\partial z}=-F_{k}$

For the lower boundary condition at the sea bottom, a zero flux of turbulent energy is assumed. Craig (1996) obtained an analytical steady-state solution of Eqs. 3 and 12 with the boundary condition (25) that can be used to derive the surface Dirichlet boundary condition for TKE (at $z=z_{s}$ ) as follows:

$k=A_{k}\left[a+c_{\mu}^{0} \beta\left(\frac{3 \sigma_{k}}{2}\right)^{0.5}\left(\frac{z_{0}-z_{s}}{z_{0}}\right)^{-m}\right]^{2 / 3}$,

where

$m^{2}=\frac{3}{2} \frac{\left(c_{\mu}^{0}\right)^{2} \sigma_{k}}{\kappa^{2}}$ and $A_{k}=\left(\frac{u_{*}^{w}}{c_{\mu}^{0}}\right)^{2}$.

In equation 26, $a=1$ and $a=0$ are used for shear flows and the pure wave breaking conditions, respectively (Burchard 2001; Carniel et al. 2009).

\subsubsection{Boundary conditions for the $\varepsilon$-equation}

To obtain the flux boundary condition for $\varepsilon$, the vertical derivative of $\varepsilon$, defined by Eq. 20, is taken as follows:

$\left[\frac{v_{t}}{\sigma_{\varepsilon}} \frac{\partial \varepsilon}{\partial z}\right]_{z=z_{s}}=\frac{v_{t}}{\sigma_{\varepsilon}}\left(c_{\mu}^{0}\right)^{3}\left[\frac{3}{2} \frac{k^{1 / 2}}{l} \frac{\partial k}{\partial z}-k^{3 / 2} l^{-2} \frac{\partial l}{\partial z}\right]$.

By defining the length scale as $l=\kappa\left(z_{0}-z\right)$, and using Eq. 25 , the flux boundary condition for $\varepsilon$ at $z=z_{s}$ becomes

$\left[\frac{v_{t}}{\sigma_{\varepsilon}} \frac{\partial \varepsilon}{\partial z}\right]_{z=z_{s}}=-\frac{3}{2} \frac{\sigma_{k}}{\sigma_{\varepsilon}} \frac{k^{1 / 2} F_{k}\left(c_{\mu}^{0}\right)^{3}}{\kappa\left(z_{0}-z_{s}\right)}+\frac{v_{t}}{\sigma_{\varepsilon}} \frac{k^{3 / 2}\left(c_{\mu}^{0}\right)^{3}}{\kappa\left(z_{0}-z_{s}\right)^{2}}$.

The Dirichlet boundary condition for dissipation rate of TKE at surface (in the presence of wave breaking) is obtained using boundary condition (25) and 22 as follows:

$[\varepsilon]_{z=z_{s}}=A_{\varepsilon}\left[a+c_{\mu}^{0} \beta\left(\frac{3 \sigma_{k}}{2}\right)^{0.5}\left(\frac{z_{0}-z_{s}}{z_{0}}\right)^{-m}\right]$,

where $m$ is defined in Eq. 27 (Burchard 2001), $a$ is introduced in Section 3.5.2, and $A_{\varepsilon}$ is

$A_{\varepsilon}=\frac{\left(u_{*}^{w}\right)^{3}}{\kappa\left(z_{0}-z_{s}\right)}$.
3.6 Water-side roughness length

Bye (1988) used Charnock-type relationship for determining the water-side roughness length:

$z_{0}=\alpha \frac{\left(u_{*}^{w}\right)^{2}}{g}$.

Churchill and Csanady (1983) suggested $\alpha=1,400$ according to the near-surface velocity measurements. In the presence of waves, based on experimental evidence, Terray et al. (1996) showed that $\alpha$ in Eq. 31 is not a constant, and proposed that $z_{0}$ should be parameterized via the significant wave height, $z_{0} \sim O\left(H_{s}\right)$. Using a data set from the Knight Inlet, Stacey (1999) obtained $\alpha \approx O\left(10^{5}\right)$ and $z_{0}=0.5 H_{s}$. Gemmrich and Farmer (1999) found that $z_{0}=0.2 \mathrm{~m}$, independent of $H_{s}$, which had a mean value of $4.5 \mathrm{~m}$ during their experiment. Soloviev and Lukas (2003) obtained $\alpha=90,000$ based on a fit between model data and field data. Stips et al. (2005) found a better agreement between model data and their observations using $\alpha=14,000$. Jones and Monismith (2007) used $\alpha=32,000$ as a suitable choice for improving their model results. Hence, the actual value of $z_{0}$, especially during strong and intense wind events, is still at debate.

Using the common practice, we use Charnock-type relationship (31) with $\alpha=1,400$ to calculate water-side roughness length for NW simulation run and apply (Terray et al. 1996) parameterization of $z_{0}$ for all simulation runs with wave forcing effects.

\section{Results and discussion}

\subsection{Atmospheric forcing}

Since the surface buoy measurements from the WS only covered a short period at the start of experiment, we use Vigra station data as the main meteorological data set for forcing the numerical runs (Fig. 2). Figure 2a, b show the wind speed and direction for the period of experiment from October 25 to 30, 2011. During the field work, the wind speed ranged from 1 to $15 \mathrm{~m} \mathrm{~s}^{-1}$ with direction typically confined within from southeast and southwest from which the wind is emanating. Two major wind events can be observed at noon of October 26 and 28 with speeds of 10.8 and $15.0 \mathrm{~m} \mathrm{~s}^{-1}$, respectively. For the remaining periods, the prevailing atmospheric conditions were calm. The air temperature was typically about $1.0-2.5^{\circ} \mathrm{C}$ warmer than the water temperature; occasionally cooler air was advected over the experiment site, especially during the two major wind events (Fig. 2d).

The surface momentum fluxes, sensible heat, and latent heat are calculated using the bulk formulae of Kondo 
Fig. 2 Time series of $\mathbf{a}$ wind speed at 10-m height, $U_{10}$, from Vigra station (solid line), and from the WS buoy (red crosses), b wind direction at 10-m height from Vigra station (solid line), and from the WS buoy (red crosses), $\mathbf{c}$ the net surface heat flux, and $\mathbf{d}$ water and air temperature at air-sea interface (black and red solid lines, respectively) for the duration of the experiment on October 25 to 30 2011. Dashed line in (c) is $Q=0$

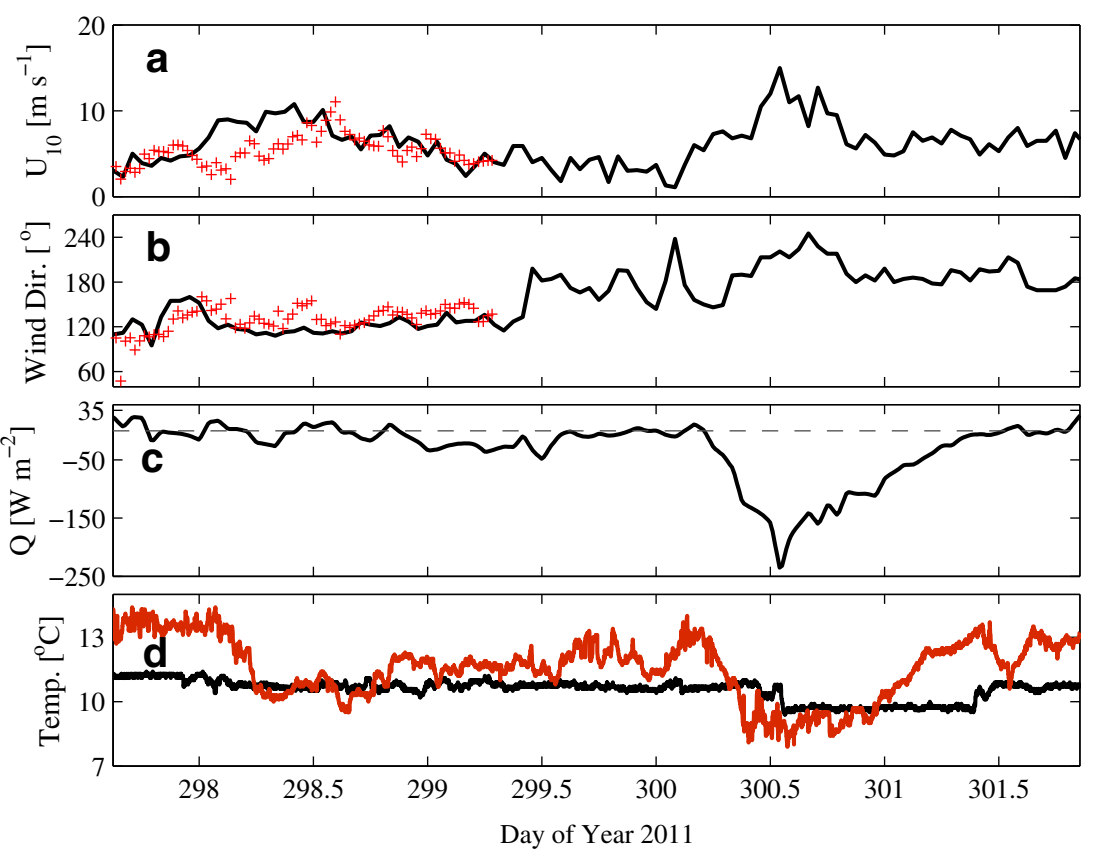

(1975). Net long wave flux is derived from the Clark et al. (1974) bulk formulae. Figure 2c shows the variability of the net heat flux into the water column. Relatively weak net surface flux conditions are interrupted substantially during the second major wind event with negative heat fluxes reaching $-250 \mathrm{~W} \mathrm{~m}^{-2}$.

\subsection{Nondirectional wave energy estimate}

To measure the nondirectional surface wave energy spectrum, we use the high-resolution pressure and motion package measurements from the MATS. The high-resolution pressure data measured in the platform body frame are first corrected for platform motions by removing the contribution from the pitch and the acceleration. The motion-corrected pressure data are then corrected for the attenuation with depth, and converted to surface wave spectra using the linear wave theory. To avoid spurious growth of noise, the high-frequency end of spectrum is extrapolated using a $f^{-5}$ power law beyond a cutoff frequency. It should be pointed out that the sea surface bulk parameters, especially those calculated from the higher order moments of the wave spectrum, are sensitive to the cutoff frequency and the parametrization of the high-frequency spectral tail.

Figure 3 shows air-side friction velocity and the comparison of significant wave height, $H_{S}$ measured by the WS and the MATS. The WS is a purpose-built wave buoy and provides accurate measurements of $H_{s}$. In general, the wave parameters estimated from MATS are in good agreement with the WS for the most of the bulk parameters, especially for the significant wave height and wave mean period,
$T_{m}$. However, estimated wave peak period shows more sensitivity and bias in some portions of the time series due to uncertainty in the extrapolation of the high-frequency tail. Figure $3 b$ shows that the temporal distribution of the significant wave height is connected to the wind forcing and local fetch effect. Early in the record, due to short local fetch and duration of wind during the first major wind event, the wave field cannot build up. Large mechanical wind energy from the strong southwestward surface wind field on day 300.5 is transferred to the wave field. This wave field developed after the second major wind event and remained strong despite the decaying wind speed.

\subsection{Wave-induced forcing}

The evolution of wave energy spectrum is shown in Fig. 4a. During the second major wind event on October 28 , an increase in the peak frequency is accompanied by an increase in the spectral level.

The Stokes drift in a deep, rotating ocean can be approximated from the one-dimensional wave energy spectrum, $S(f)$ :

$\mathbf{u}_{s}^{1 D}=2 \int_{0}^{\infty} \omega k S(f) e^{-2 k|z|} d f$,

where $\omega$ is angular frequency, and $k$ is horizontal wavenumber. The Stokes drift evolution shows a high correlation with the surface gravity wave variations in the equilibrium range, especially when the large mechanical wind energy is transferred to surface waves on October 28 (Fig. 4b). During the second major wind event on day $300.6, \mathbf{u}_{s}^{1 D}$ 
Fig. 3 The time series of a the air-side friction velocity measured at the meteorological station, Vigra, and $\mathbf{b}$ significant wave height, $H_{s}$, inferred from MATS (black), and directly measured by the wave buoy WS (red crosses)

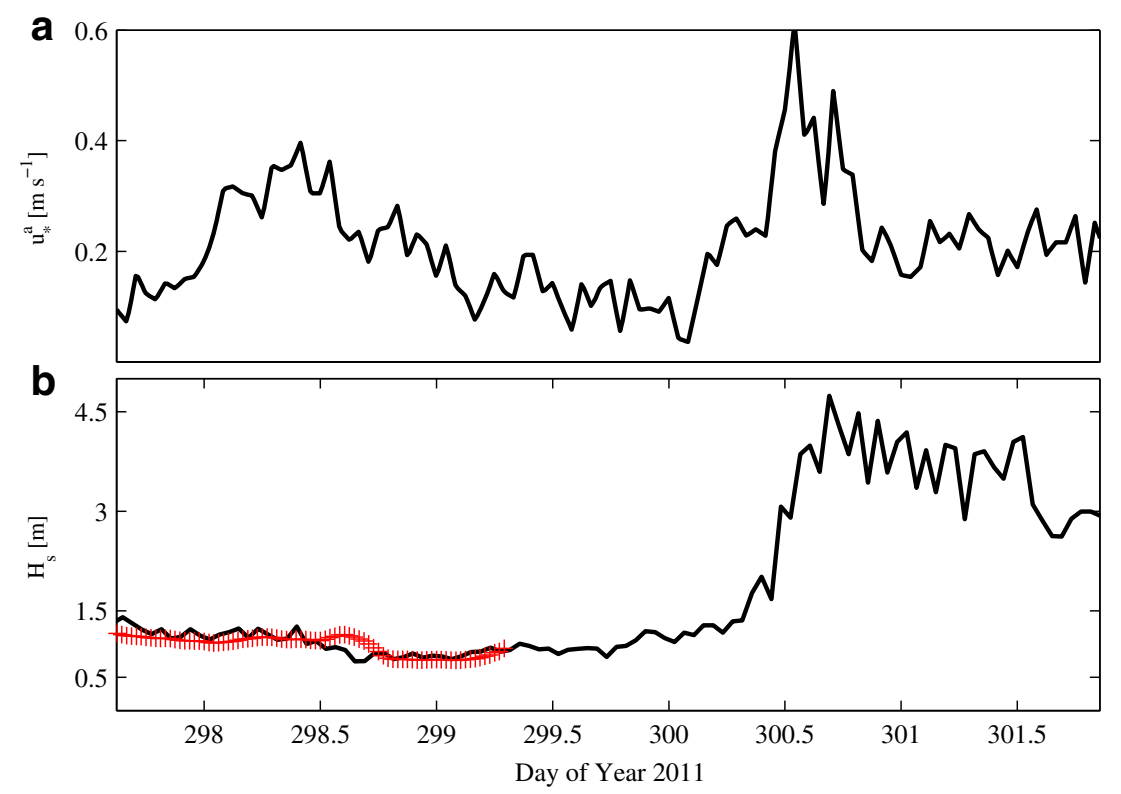

increases because of substantial contributions of the higher frequency components of the gravity waves. The vertical distribution of the Stokes drift into the deeper water is associated with the lower frequency components of the wave energy spectrum. Figure 5 shows the time series of the wind stress components, $\tau_{\text {wind }}=\left(\tau_{w}^{x}, \tau_{w}^{y}, 0\right)$, inverse of wave age, and the Stokes drift speed at the sea surface. During the windy period, the Stokes drift speed reaches $0.5 \mathrm{~m} \mathrm{~s}^{-1}$. The larger wind speed values are usually associated with younger waves (small values of $c_{\text {phase }} / u_{*}^{a}$ ), as shown in Fig. 5c, a. The importance of Langmuir circulation (LC) on producing turbulence can be inferred from the turbulent Langmuir number, as follows:

$L a_{t}=\left(\frac{u_{*}^{w}}{\left|\mathbf{u}_{s}\right|}\right)^{0.5}$.

For $L a_{t}<0.3$, LC effects become significant. During the major wind event on day $300.5, L a_{t}$ is typically between 0.1 and 0.4 , indicating that LC effects are significant enough to enhance upper ocean mixing (Fig. 5d). Note that this parameter is inversely correlated to the Stokes drift $e$-folding depth (compare Figs. 4b and 5d).
Fig. 4 Evolution of a the wave energy spectra calculated from the MATS's corrected pressure sensor in units of $\mathrm{m}^{2} \mathrm{~Hz}^{-1}$, and b the Stokes drift in $\mathrm{m} \mathrm{s}^{-1}$. Both parameters are shown in the logarithmic scale

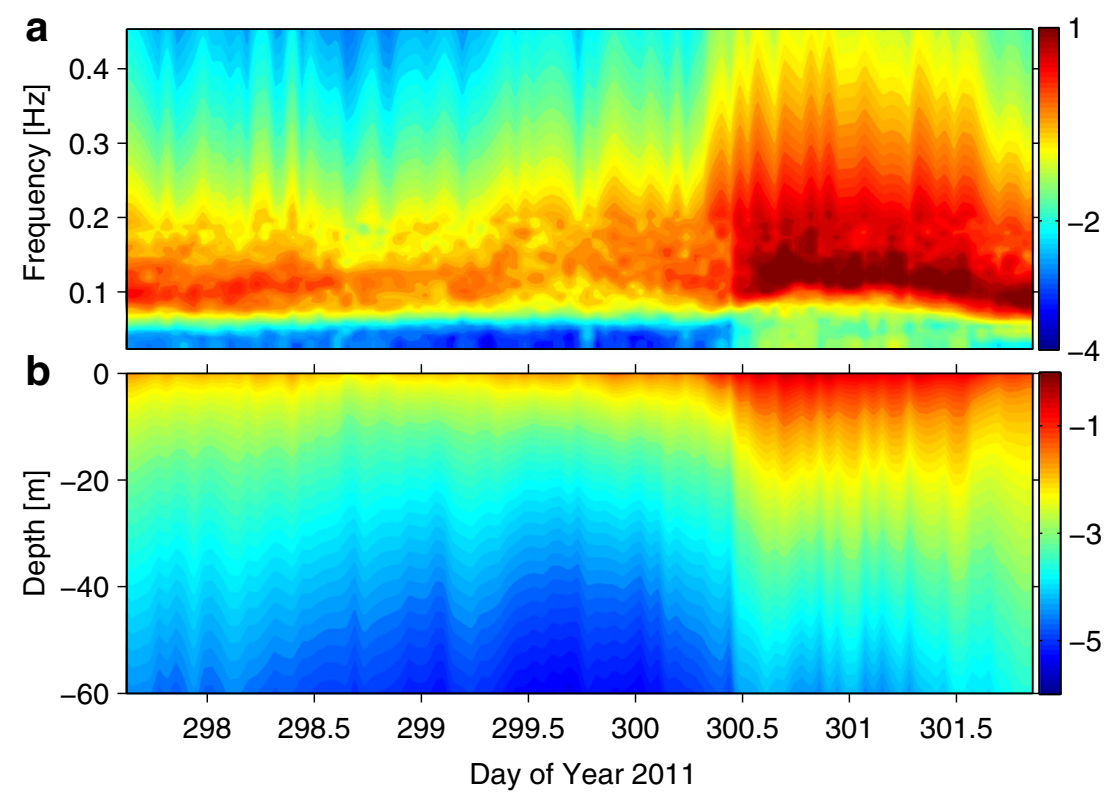


Fig. 5 Time series of a the wind stress components $\tau_{w}^{x}$ and $\tau_{w}^{y}$, measured at the Vigra station, b the surface Stokes drift speed, $\mathbf{c}$ inverse of wave age, and $\mathbf{d}$ the Langmuir turbulence number, $L a_{t}$

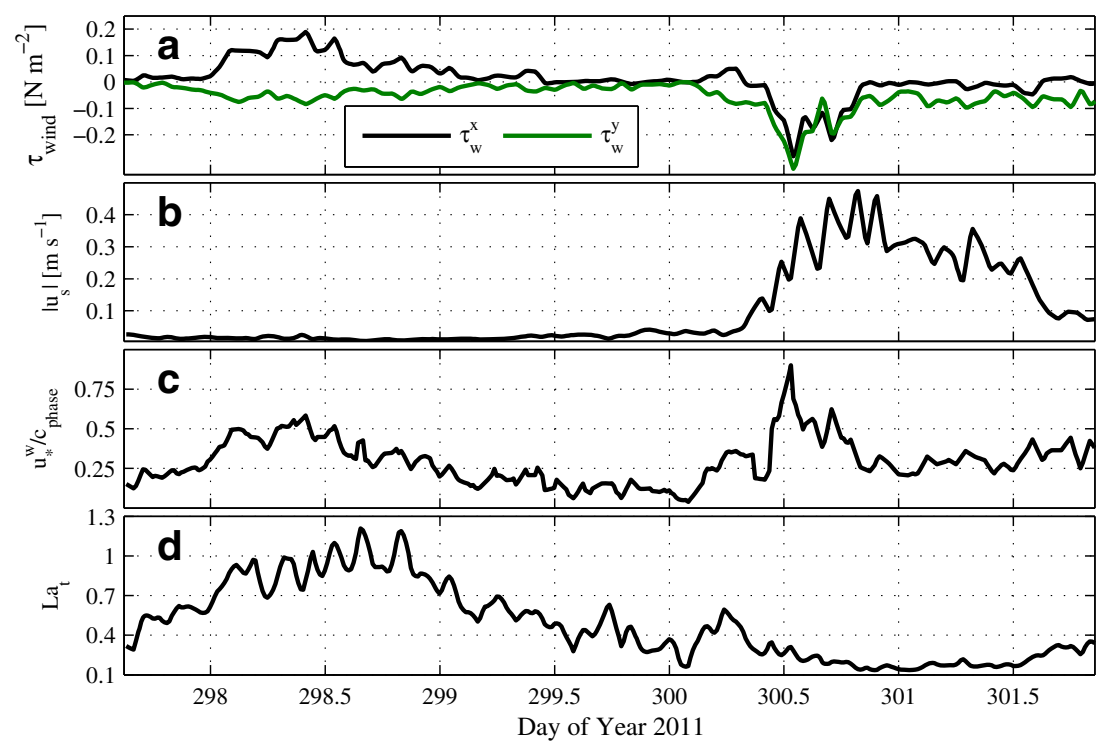

4.4 The effect of surface waves on dissipation measurements by MATS

According to the linear wave theory, the horizontal and vertical components of wave orbital velocities at depth $z$ are given as follows:

$\tilde{u}(z, t)=\sum_{m=1}^{n} \sigma_{m} \frac{\cosh \left[k_{m}(z+d)\right]}{\cosh \left[k_{m} d\right]} \eta_{m}(t)$,

and

$\tilde{w}(z, t)=\sum_{m=1}^{n} \sigma_{m} \frac{\sinh \left[k_{m}(z+d)\right]}{\sinh \left[k_{m} d\right]} \tan \left(\psi_{m}\right) \eta_{m}(t)$,

where $d$ is the total water depth, $\psi_{m}=\sigma_{m} t+\phi_{m}$, and $n$ is the total number of wave components. In the absence of Doppler shifting of wavenumber $k_{m}$, the radian frequency $\sigma_{m}$ is prescribed by dispersion relation, as follows:

$\sigma_{m}^{2}=g k_{m} \tanh \left(k_{m} d\right)$,

where $m=1, \ldots, n$ and $\eta_{m}(t)=a_{m} \cos \left(\sigma_{m} t+\phi_{m}\right)$ is the $m$ th component of sinusoidal wave with amplitude $a_{m}$, and random phase $\phi_{m}$.

In Fig. 6, the frequency spectra from the shear probes are compared to the equivalent spectrum of shear induced by the vertical component of wave orbital velocity, $\tilde{w}$, at the measurement depth. Spectra of the orbital horizontal components are approximately equal to that of $\tilde{w}$, and are not shown. There is a significant variance in the wave frequency range, in this burst with a peak at 10-s period, which rapidly decays with frequency. The contribution of the wave-induced shear to the turbulence range used in this study is negligible. The time series from the second shear probe, $\mathrm{SH} 2$, consistently shows suppressed variance in the wave range and elevated variance in the turbulence range.
The shape and amplitude of the spectra for $\mathrm{SH} 2$ cannot be explained in the light of motion and vibration sensors, and we discard $\mathrm{SH} 2$ from the analysis. The spectra from the accelerometer data and the vibration sensor are rescaled to the equivalent units of shear and represent the variance in the low frequency wave band (GAz) and in the highfrequency range ( $>1 \mathrm{~Hz}, \mathrm{VAz}$ ), respectively. Here, only the vertical components (GAz and VAz) are shown; however, the spectra from the other components are similar. There is significant acceleration in the wave frequency range, on the same order as the shear probe signal. The shear probe signal in the frequency band $1-20 \mathrm{~Hz}$ is less affected by the wave motions (typical of all bursts), and the high-frequency vibration. In calculation of the dissipation rates, the frequency range between 1 and $20 \mathrm{~Hz}$ is used. This ensures that the signal is not corrupted by the substantial variance induced by the wave orbital velocities, the low-frequency platform motion, and the high-frequency noise due to vibration.

\subsection{Comparisons between model runs and observations}

The one-dimensional mixed layer GOTM system based on the $k-\varepsilon$ type second moment closure of turbulence is used to study wave forcing effects on the upper ocean turbulence variations. The model is set up at the location of MATS, and it is assumed that all measured data used to force the model are representative of this location. We use a temporal resolution of $\Delta t=10 \mathrm{~s}$, and a vertical nonequidistance resolution with a slight zooming to the surface. In total, three runs were made, no wave (NW) run, and two runs with different wave forcing terms (W1 and W2) summarized in Table 3. It should be noted that in both wave-forced simulation runs, we use the wave-induced momentum redistribution term (6) and do not 

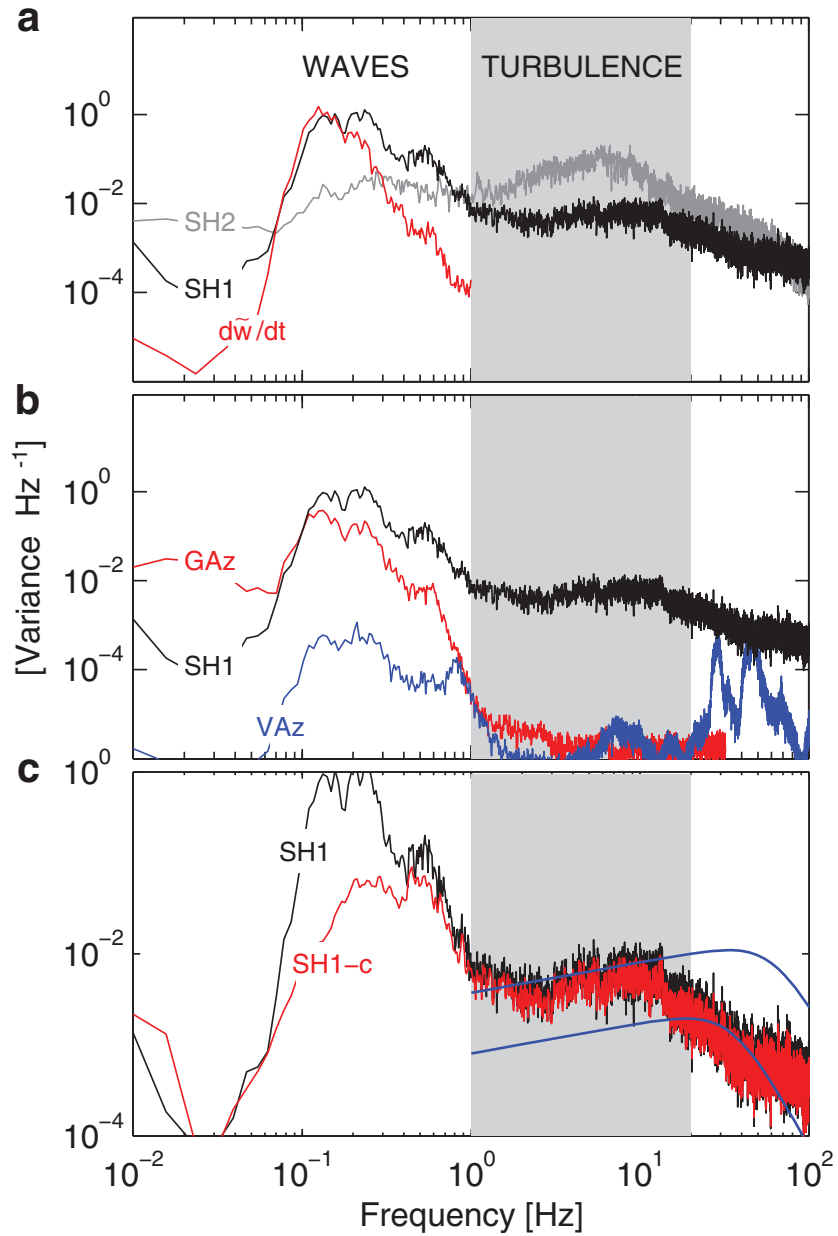

Fig. 6 Frequency spectra for a 15-min burst recorded on 26 October $2011,12: 08$ UTC. a Spectra inferred from shear probes $(d w / d t$, SH1, and $d v / d t, \mathrm{SH} 2$ ) and the equivalent wave orbital velocity spectrum, $d \tilde{w} / d t$, inferred from irrotational wave theory using the measured surface wave spectrum. b Spectra from shear probe SH1 compared with the vertical acceleration spectra from the gyro $(\mathrm{GAz}$, representative for motion below $1 \mathrm{~Hz}$ ) and the vibration sensor (VAz, representative of motion above $1 \mathrm{~Hz}$ ). c Spectra from shear probe before (SH1) and after (SH1-c) removing the coherent acceleration signal using the Goodman et al. (2006) algorithm. Also shown are the empirical Nasmyth's spectra for $\varepsilon=10^{-6}$ and $10^{-5} \mathrm{~W} \mathrm{~kg}^{-1}$. The shaded parts in each panel mark the frequency range used in calculating the dissipation rate from the shear probes, unaffected by the wave motion

use the wave breaking production term (vertical derivative of Eq. 13), which are not listed in Table 3.

Kantha and Clayson (2004) included LC in turbulence closure models by adding the Stokes drift production term to the TKE and length scale equation, and recently Harcourt (2013) extended the model of Kantha and Clayson (2004) to include Langmuir turbulence effects in the algebraic Reynolds stress model resulting in modification of stability functions and the turbulent flux closure. In this study, we examine two types of modifications for estimating wave-turbulence interaction term. First, we choose Stokes production term introduced in Eq. 17 using eddy viscosity
Table 3 Numerical run details

\begin{tabular}{lllll}
\hline Name & SP & SP & CSF & WB Eqs. \\
& Eq. 37 & Eq. 17 & Eq. 4 & 25 and 29 \\
\hline NW & No & No & No & No \\
W1 & No & Yes & Yes & Yes \\
W2 & Yes & No & Yes & Yes \\
\hline
\end{tabular}

The run without wave forcing is NW. Two simulation runs with wave forcing (W1 and W2 runs, respectively) include Stokes Production (SP), CSF, and Wave Breaking (WB) effects as listed

relation 19. Secondly, we use the parametrization introduced by Huang and Qiao (2010). In Eq. 12, we assume the dissipation rate is balanced by the production terms in the TKE equation as $\varepsilon=P_{\text {curr }}+P_{\text {Stokes }}$.

Following Huang and Qiao (2010), $P_{\text {Stokes }}$ is replaced by $a_{1}\left(u_{*}^{w}\right)^{2}\left|\frac{\partial \mathbf{u}_{s}}{\partial z}\right|$,

where $|\cdot|$ denotes the magnitude of vertical gradient of Stokes drift vector, and $a_{1}$ is a nondimensional constant associated with the surface gravity waves which can be prescribed by regression against the observations of $\varepsilon$ collected by Anis and Moum (1995) as follows:

$a_{1}=3.75 \beta^{\prime \prime} \pi \sqrt{\frac{H_{s}}{\lambda}}$,

where $\lambda$ is wavelength and $\beta^{\prime \prime}$ is a dimensionless constant between 0 and 1. Huang and Qiao (2010) validated the skill of their parametrization by comparing to the observations of Anis and Moum (1995), Wüest et al. (2000), and Osborn et al. (1992). Figure 7 shows the time evolution of estimated $a_{1}$ during the course of our experiment with values ranging between 0.6 and 2.4. As expected, there is a high correlation between the significant wave height and $a_{1}$.

The observed temperature, $T$, is assimilated into the model in the upper $60 \mathrm{~m}$ of the water column, using a relaxation time scale of $12 \mathrm{~h}$. Temperature record at mooring $M$ shows a thermally stratified mixed layer for the whole campaign. Oscillation of temperature is modulated by vertical pycnocline motions at the base of mixed layer, with the heat fluxes into the water and major wind events (Fig. 8b). The resulting temperature field for the NW run is shown in Fig. 8a, compared with the observed temperature (Fig. 8b). Although this good agreement between the modeled and observed temperature is expected because of the relaxation, the assimilation is crucial to successfully model the turbulence variability near the sea surface.

Figure 9 compares the depth-time evolution of the observed and modeled TKE dissipation rate for NW, W1, and $\mathrm{W} 2$ runs. It should be noted that the use of a tethered free-falling profiler cannot make it possible to measure 
Fig. 7 Time series of the dimensionless parameter $a_{1}$ (black), and the significant wave height, $H_{s}$ (red dashes)

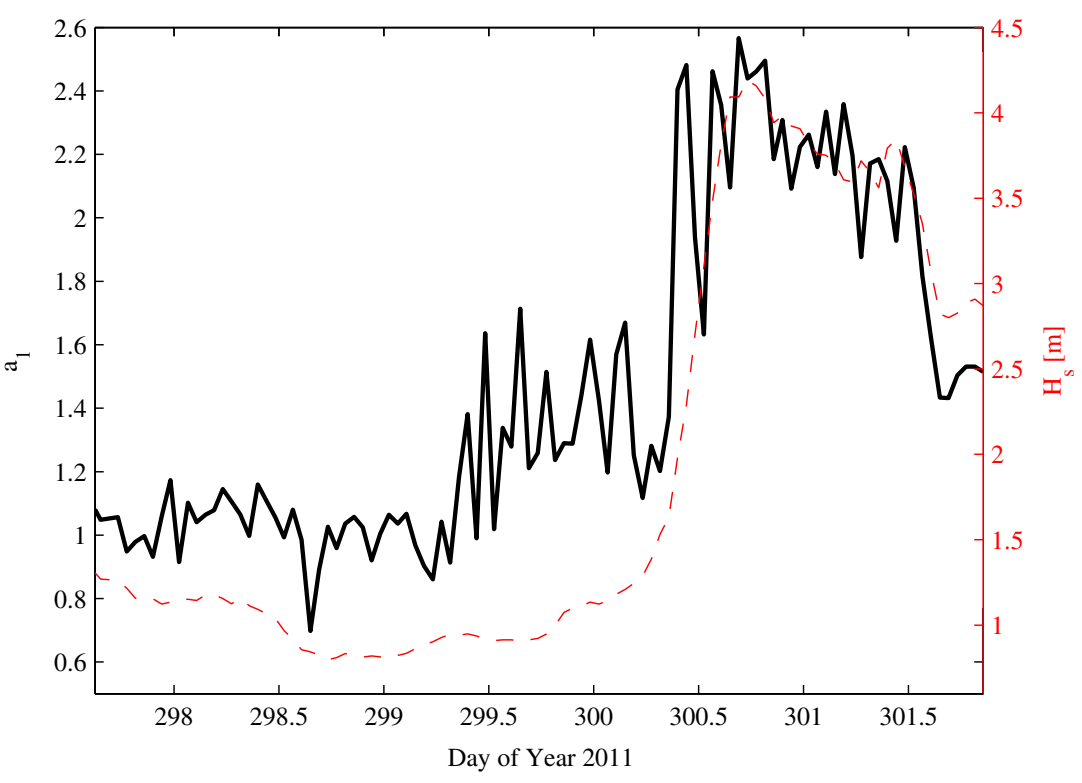

the dissipation rate accurately near the sea surface due to contaminations induced by the ship's wake, wave orbital velocities, the oscillations, and vibrations induced by the tether. Thus, we confine our comparisons near the sea surface for depths between 5 and $60 \mathrm{~m}$. The dissipation profile measurements are limited to four short periods (Table 1). The periods when the strong wind and waves can be expected to produce the highest TKE injection into the nearsurface water column were not sampled. Measurements of $\varepsilon$ show small-scale variability that may be attributed to the intermittency of turbulence (Fig. 9). Elevated values of $\varepsilon$ penetrate deep into the water column following the period with increased surface stress.
Figure 9d, e show the model simulation results for the dissipation rate of TKE with wind and wave forcing. Because of the sparse sampling during the experiment, we cannot conclude on the skill of the GOTM wave modifications to reproduce the dissipation rates realistically. Nonetheless, enhanced dissipation rates near the surface, especially by the end of experiment after the major wind event on October 28, are notable features in both observations and model simulation results. Figure 9d, e also show that the enhanced dissipation rates occur not only near the sea surface due to breaking waves, but also throughout the active-mixed layer. In the absence of wave effects during wind events, the simulation predicts weaker $\varepsilon$ (Fig. 9c).
Fig. 8 Depth-time evolution of a modeled temperature, and $\mathbf{b}$ the observed temperature field for the entire duration of experiment. The color bar is in ${ }^{\circ} \mathrm{C}$ and the contours are drawn at $0.05{ }^{\circ} \mathrm{C}$ intervals. The model results are from run $\mathrm{W} 2$; the observations are from moored instruments at $\mathrm{M}$, averaged in hourly intervals

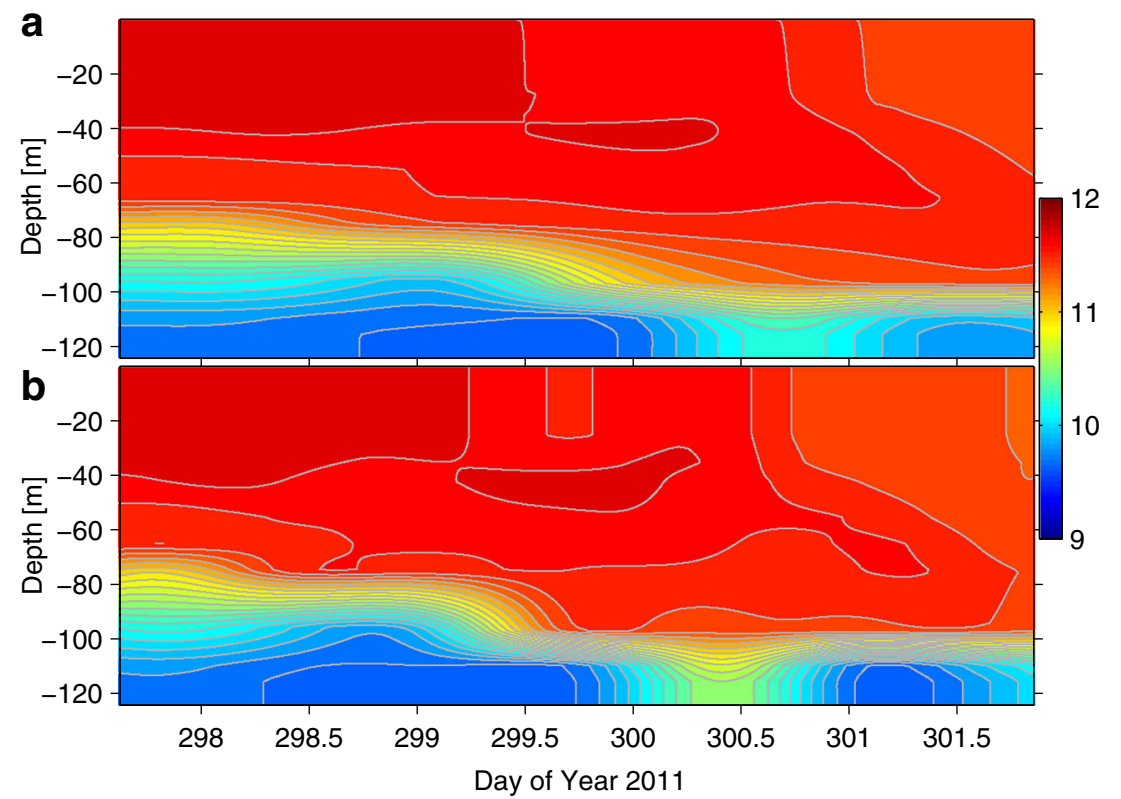


Fig. 9 Time series of a air-side surface friction velocity, and depth-time evolution of $\mathbf{b}$ the dissipation rate of TKE measured by the MSS profiler, simulated in runs $\mathbf{c} \mathrm{NW}, \mathbf{d} \mathrm{W} 1$, and $\mathbf{e} \mathrm{W} 2$. All dissipation rates are given in $\mathrm{W} \mathrm{kg}^{-1}$ and in logarithmic scale

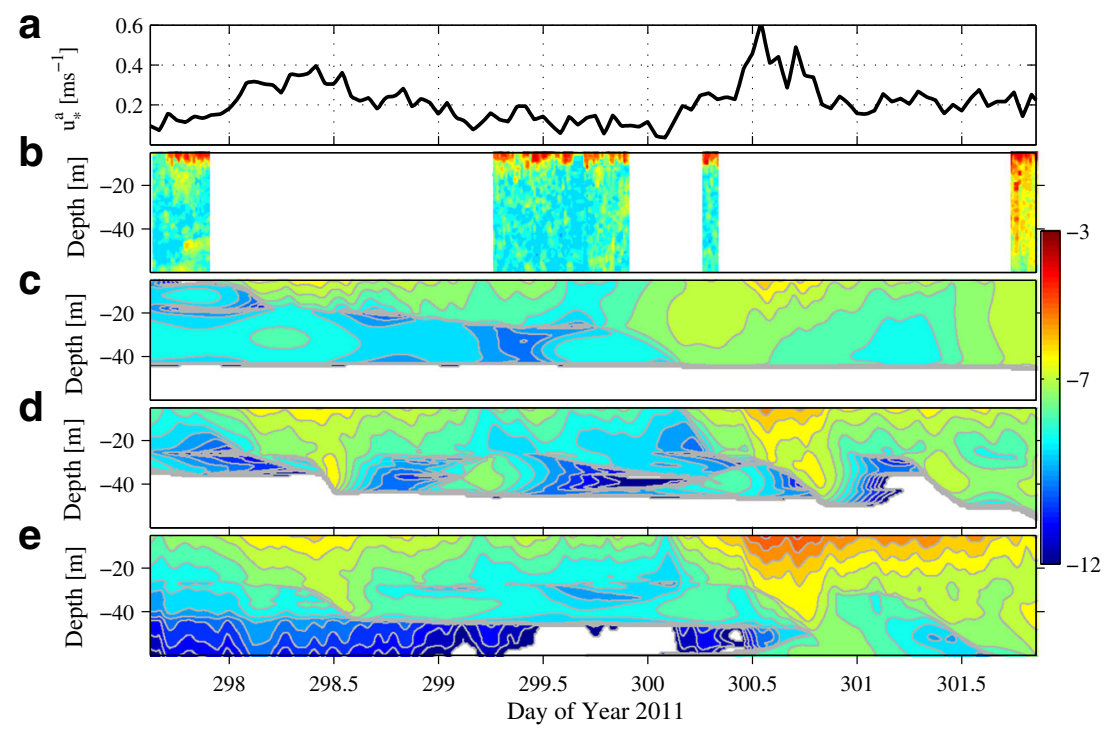

Including the wave effects, especially in the W2 run, results in a strong regulation of the vertical mixing near the sea surface, and produces deeper penetrating enhanced dissipation rates relative to the $\mathrm{W} 1$ simulation. The covariability of $\varepsilon$ with the air-side friction velocity shows that the effects of wave-current-turbulence depend on the wind stress magnitude. During calm conditions on days between 299 and 300, the Stokes production of TKE plays an insignificant role in the regulation of the vertical TKE budget. Under the high wind and wave conditions on October 28 , the Stokes production term dominates the shear production, and $\varepsilon$ increases throughout the actively mixing layer.

At the sea surface, the eddy viscosity, $v_{t}$, scales with the air-side friction velocity and the water-side roughness length. In the deeper parts of water column, it is connected to the stratification, turbulence, and mean current variations in a more complex way. Figure 10 shows the relationship between wind and $v_{t}$ for no wave and with wave simulation runs. The eddy viscosity, as expected, is small near the sea surface during the low wind periods, and increases substantially during high wind events. Under wave forcing, the vertical distribution of eddy viscosity is highly correlated to the wavelength. Furthermore, in the period with less energetic winds and waves, days between 299.5 and 300.2, the NW simulation run is roughly proportional to wind forcing, whereas including waves increases $v_{t}$ in accordance with the temporal evolution of surface gravity wavelength. These vertical and temporal behaviors of $v_{t}$ in the presence of surface gravity waves suggest that one can estimate the eddy
Fig. 10 Time series of a smoothed wavelength (black) and air-side friction velocity (red), and depth-time contours of modeled eddy viscosity from b NW, c W1, and d W2. All modeled eddy viscosities are shown in logarithmic scale in units of $\mathrm{m}^{2} \mathrm{~s}^{-1}$

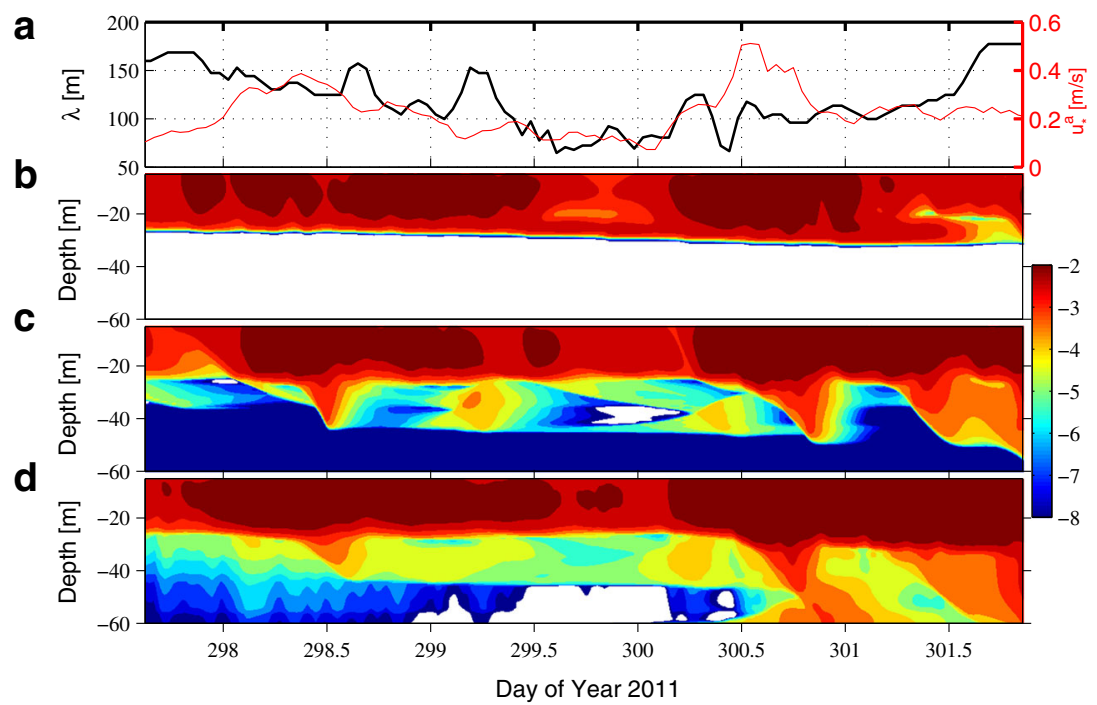


viscosity near the sea surface using dimensional analysis as,

$v_{t} \propto u_{*}^{w} l_{w}$

where $l_{w}$ is a wave-induced length scale proportional to the wavelength $\lambda$. Additionally, Qiao et al. (2004) proposed that this wave-induced length scale is proportional to the wave particle displacement. Meanwhile, they used this length scale to produce an additional wave-induced vertical kinetic viscosity component, $B_{v}=\overline{l_{w} \tilde{w}}$ where $\tilde{w}$ denotes vertical component of wave-induced fluctuation to the conventional eddy viscosity expression. Using linear wave theory, they obtained:

$$
\begin{aligned}
B_{\nu}(z)= & \alpha_{\nu} \iint S(\mathbf{k}) \exp (-2 k|z|) d \mathbf{k} \frac{\partial}{\partial z} \\
& {\left[\iint \omega^{2} S(\mathbf{k}) \exp (-2 k|z|)\right]^{\frac{1}{2}}, }
\end{aligned}
$$

where $S(\mathbf{k})$ denotes the wave energy spectrum in the wavenumber vector space, $\mathbf{k}=k \hat{\mathbf{k}}$, and $\alpha_{v}$ is a calibration parameter. Comparing the modified eddy viscosity (and modified eddy diffusivity) effects on the numerical simulation of upper ocean turbulence variability with those obtained by NW, W1, and W2 runs is not the scope of current study. Therefore, we do not provide such comparisons here.

Production of turbulence was dominated by wind and wave forcing throughout the experiment. During the period of strong cooling on October 28, the TKE production by shear was several orders of magnitudes greater than the production due to buoyancy flux. In all other MSS profiling periods, the water column was statically stable. The vertical distribution of the gradient Richardson number $(R i=$ $N^{2} M^{-2}$ ) in the water column suggests that the background mean shear was not strong enough to contribute to the turbulence production, except during the last set of measurements when the water column was marginally stable for shear instability. $R i$ is calculated using the squared buoyancy frequency, $N^{2}$, from temperature and salinity profiles measured by the MSS, and squared shear, $M^{2}$, from an the uplooking ADCP measurements located at $80 \mathrm{~m}$ below the sea surface. The values of $N^{2}$ and $M^{2}$ are temporally averaged at each of four MSS sets. Figure 11 indicates generally that the observed mean current shear during the course of experiment cannot account for the elevated levels of turbulence evident in the observations and the modeling results.

Figure 12 shows the scatter plot of dissipation rate of TKE measured at 8-m depth versus wind speed at $10-\mathrm{m}$ height. Observed $\varepsilon$ from MATS and MSS are reasonably correlated with each other (with a correlation coefficient of 0.62) and correlate monotonically with the wind speed. Moreover, the dissipation rates cover values from $3 \times 10^{-9} \mathrm{~W} \mathrm{~kg}^{-1}$ during the low wind speed conditions to $10^{-5} \mathrm{~W} \mathrm{~kg}^{-1}$ in windy conditions. The minimum $\varepsilon$ measured by MSS is about $3 \times 10^{-9} \mathrm{~W} \mathrm{~kg}^{-1}$ which is near the noise level for this instrument. The lowest value of dissipation rates inferred from the MATS shear probes is about $2 \times 10^{-8} \mathrm{~W} \mathrm{~kg}^{-1}$, suggesting that MATS shear probes for measuring dissipation rates may have higher noise level than the MSS shear probes.

In Fig. 9b, the nature of observed dissipation rates demonstrates strong intermittency. The strong variability of
Fig. 11 Time averaged vertical profiles of squared shear, $M^{2}$ (black), squared buoyancy frequency, $N^{2}$ (red), and $R i=N^{2} / M^{2}$ (gray): a periods between days 297.5 and 297.8 , b 299.4 and 299.9 , c 300.35 and 300.4, and $\mathbf{d} 301.8$ and 302. Vertical dashed line marks $R i=1$. The vertical gradients in $M^{2}$ and $N^{2}$ are calculated using 2-m vertical separation

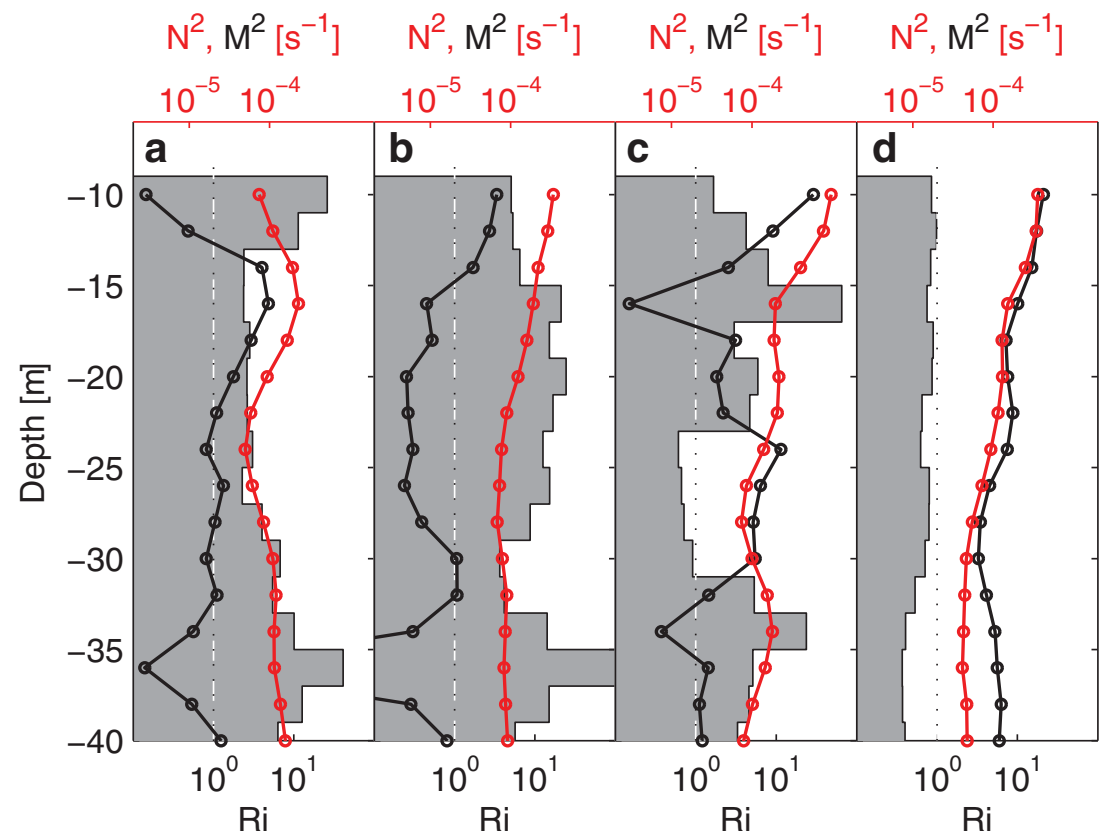


Fig. 12 Mean dissipation rate at a depth of $8 \mathrm{~m}$ below the sea surface measured by MATS (squares), and measured by the MSS profiler (circles), versus wind speed at $10-\mathrm{m}$ height measured at the Vigra station

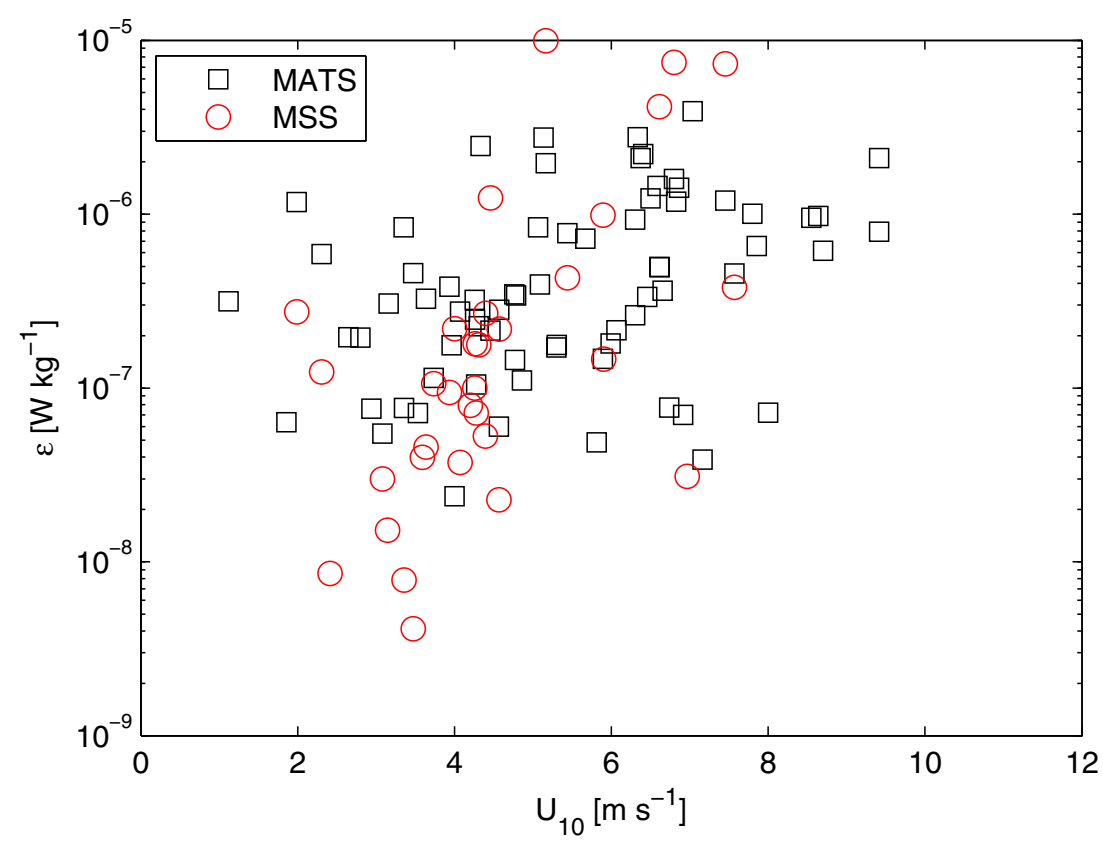

$\varepsilon$ near the sea surface in the response to the change of wind speed and direction necessitates an appropriate way to average such an intermittent and complex variable. Following Baker and Gibson (1987), we use a maximum likelihood estimate (MLE) for the mean dissipation rate of TKE. In the case of $\log$-normal distribution of $\varepsilon$, the MLE mean value is

$<\varepsilon>_{M L E}=\exp \left(\mu+\frac{\sigma^{2}}{2}\right)$,

where $\mu$ is the arithmetic mean, and $\sigma^{2}$ is the variance of $\ln (\varepsilon)$.

In the case of model simulation results, the arithmetic mean value is a good estimator for the expected value of $\varepsilon$. To investigate depth dependence of dissipation rates of TKE in more detail, we present MLE profiles of $\varepsilon$ measured by the MSS and MATS for the four identified periods of MSS profiling. Figure 13 shows the set averaged, measured, and modeled profiles of $\varepsilon$. A larger variability close the sea surface can be seen in response to the wind and wave forcing. The W2 modeled profiles of $\varepsilon$ agree with observations fairly well, suggesting that the shear generated by the Stokes drift is a likely mechanism for enhancement of $\varepsilon$ in the water column away from breaking-wave affected zone. However, under the low wind speed, observations of $\varepsilon$ show unexpected quite high turbulence levels (Fig. 13). Generally, a good agreement is seen between the MATS-inferred $\varepsilon$ and the dissipation rate measured by the MSS. A comparison of profiles of $\varepsilon$ and $R i$ (shown in Fig. 11) suggests that $\varepsilon$ increases near the surface with decreasing values of $R i$.

Averaging in time may smooth out the changes in the structure of turbulence in varying wave forcing conditions.
To delineate the influence of wave forcing, we select ensembles of profiles during: low $\left(H_{S} \leq 1 \mathrm{~m}\right)$, moderate $(1<$ $\left.H_{s}<2 \mathrm{~m}\right)$, and high wave $\left(H_{s} \geq 2 \mathrm{~m}\right)$ conditions. Ensemble-averaged $\varepsilon$ profiles for each case along with ensemble average of wave energy spectrum are shown in Fig. 14. Under all wave conditions, $\varepsilon$ measured by the MATS and by the MSS at the corresponding depth agree within error bars. Because of removing the larger percentages of points measured by MSS near the sea surface, the confidence intervals (gray shaded regions) are larger near the sea surface than those in the deeper part of water column. Moreover, the agreement between measurements and model runs is better when wind and wave activities are accounted for all three wave periods.

Figure 15 shows a direct comparison of time series $\varepsilon$ measured by the MATS's shear probe, and by the MSS profiler, $\varepsilon_{M S S}$, averaged at the depth of MATS, as well as $\varepsilon$ extracted from the model simulations. Time series are averaged in 1-h intervals (using MLE). Estimates of $\varepsilon_{M S S}$ are averaged in the vertical within $2 \mathrm{~m}$ centered at the depth of MATS. Estimates of dissipation rates from MATS are shown with their corresponding $95 \%$ confidence intervals. Error bars are relatively small except at a period before the fetch-limited wind event on October 26, and also before the major wind event between days 299 and 300. The two independent observations of $\varepsilon$ are fairly correlated, except for the period at the end of experiment, and for a period when the wind speed dropped to below $2.5 \mathrm{~m} \mathrm{~s}^{-1}$. Furthermore, model results from $\mathrm{NW}, \mathrm{W} 1$, and $\mathrm{W} 2$ simulations are compared with the measured dissipation rates of TKE. Model results predict the dissipation rate enhancement of several orders of magnitude, few meters below the sea 
Fig. 13 Profiles of dissipation rates in logarithmic scale for the four different sets of MSS profiling periods during October 2011. Shown are the results from the simulations by NW (solid lines), simulations by $\mathrm{W} 1$ (dashed lines), simulations by W2 (bold solid lines), the observations made by MSS profiler (red solid lines), and the observed data from MATS at fixed depth (square markers) The shaded gray regions denote the confidence intervals of measured $\varepsilon$ from MSS profiler

Fig. 14 Dissipation rates of TKE profiles in logarithmic scale for the three different wave conditions. Confidence intervals for MSS measurements of $\varepsilon$ are denoted by the shaded gray regions. Shown are the results from the simulations NW (solid lines), W1 (dotted lines), and W2 (thick solid lines), the observed data from MSS profiler (red solid lines), and MATS at a fixed level (square markers) for a calm sea state, $\mathbf{b}$ moderate waves, and $\mathbf{c}$ high waves. Lower panels show the wave energy spectrum, $S_{\eta}$, estimated using pressure sensor mounted on MATS for $\mathbf{d}$ calm sea state, e moderate waves, and f high waves
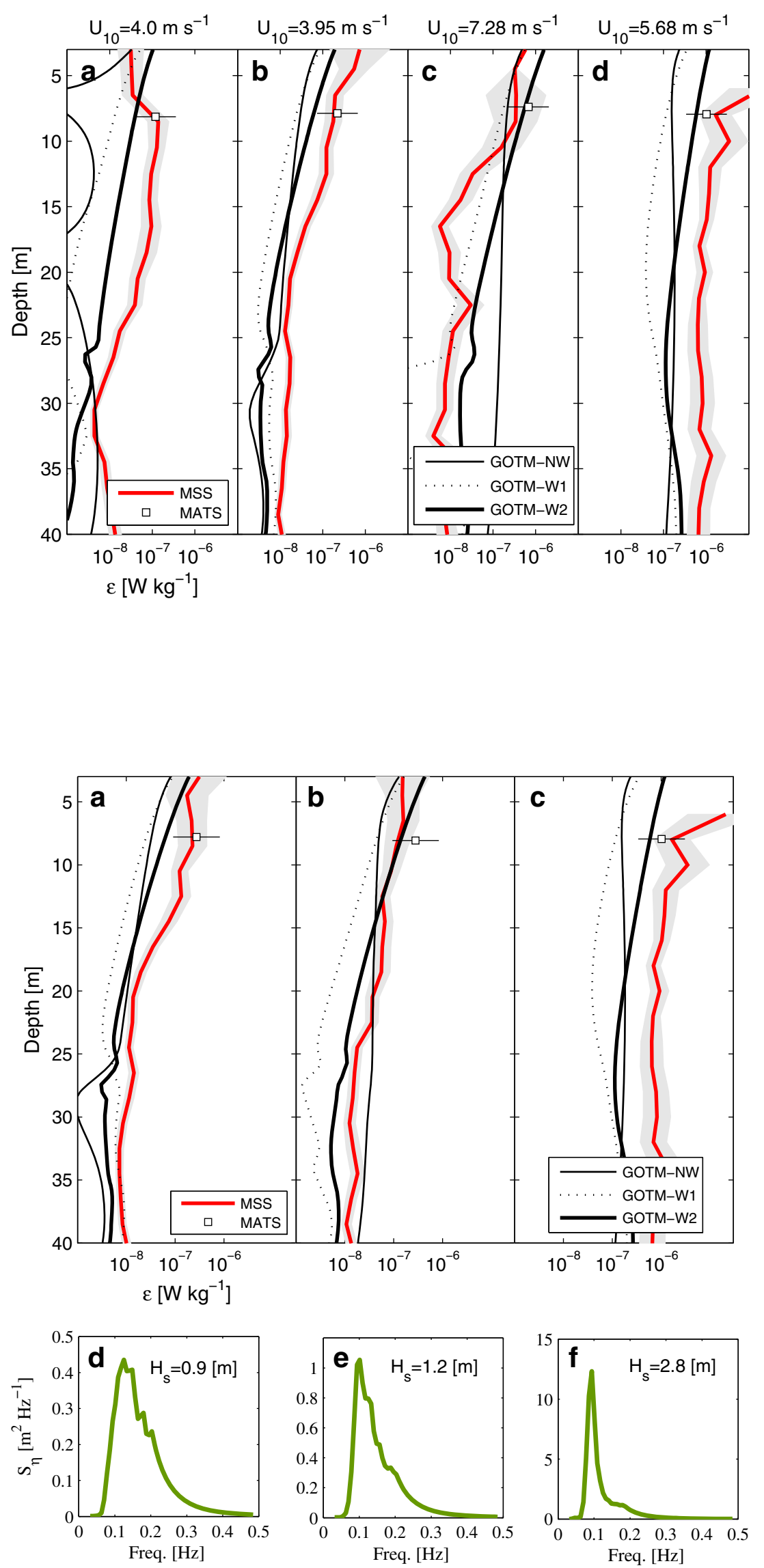
Fig. 15 Mean dissipation rate time series at depth $8 \mathrm{~m}$ below the sea surface measured from MATS, measured from MSS profiler, runs by NW, W1, and W2, and estimate from TA96, with markers and lines as indicated in the legend

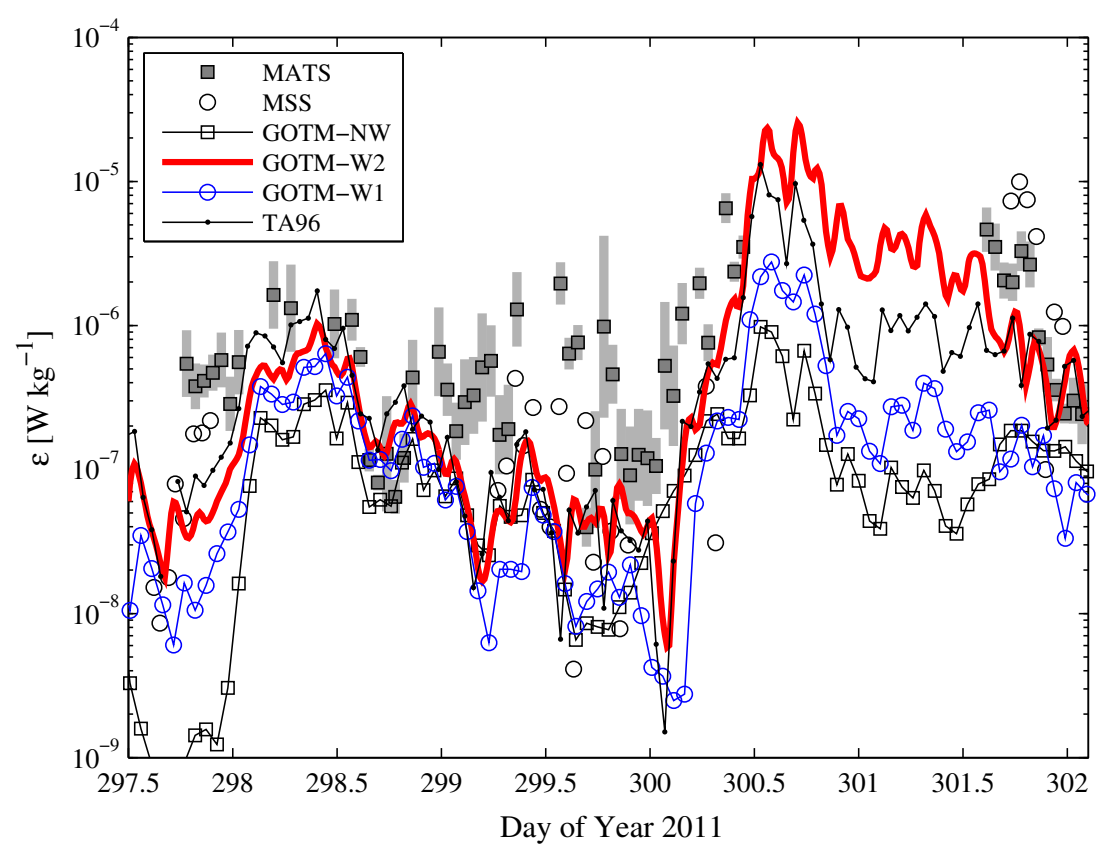

surface due to wave breaking (not shown here). This enhancement clearly extends to our measurement depth of about $8 \mathrm{~m}$ below the sea surface, mainly due to the increase of shear stress by the wave-induced shear production of TKE. The NW results underestimate the observed $\varepsilon$, especially during the major wind events. Although both W1 and W2 results improve prediction of dissipation rates during the two major wind events, they cannot capture the turbulence variability at the depth of MATS for a period when the wind speed dropped below $2.5 \mathrm{~m} \mathrm{~s}^{-1}$. Comparisons of modeled and observed $\varepsilon$ reveal large deviations which can be attributed to intermittency, statistics, and platform motion-induced contaminations.

The modeled and observed estimates of $\varepsilon$ are further compared with the empirical prediction of Terray et al. (1996) extracted from the deep water wave breaking scaling as (hereafter referred as TA96)

$\frac{\varepsilon H_{s}}{\left|F_{k}\right|}=0.3\left(\frac{z}{H_{s}}\right)^{-2}$,

where $F_{k}$ is the breaking wave-induced flux of TKE into the water column defined in Eq. 22 using $\beta=100$. Generally,
Fig. 16 Profiles of dissipation rate in logarithmic scale normalized following TA96, for the four different sets of profiling periods during October 2011 (Table 1). The black lines denote TA96 estimated dissipation rates profiles, the dashed black lines are the model predictions by Burchard and Bolding (2001), and the solid, dotted, and dash-dotted blue lines are $\mathrm{NW}, \mathrm{W} 1$, and $\mathrm{W} 2$ simulation results, respectively. Bold circle, green square, and red triangle markers are (Gerbi et al. 2009), MATS, and MSS nondimensional $\varepsilon$ data
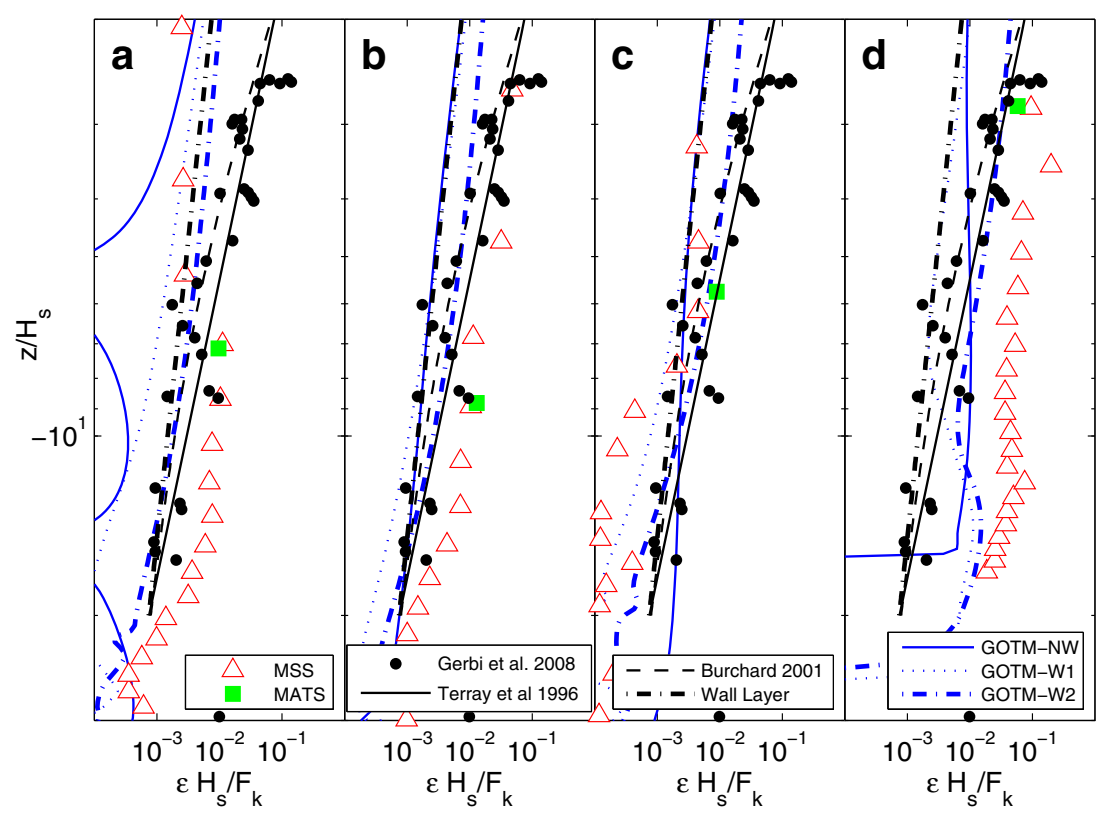
comparisons show that the TA96 estimates of $\varepsilon$ agree fairly well with W1 and W2 runs, especially during the strongest wind event (Fig. 14).

The near-surface observed and modeled dissipation rate profiles are scaled using $H_{s}$ and $F_{k}$ as suggested by TA96. In Fig. 16, data and model results from this study are compared to the published observations carried out by Gerbi et al. (2009), as well as model dissipation rates from Burchard (2001). The normalized profile of $\varepsilon$ from MSS agrees well with those from (Gerbi et al. 2009) and TA96. Meanwhile, in all MSS profiling periods, there is a good agreement among $\varepsilon$ derived from the three different techniques and instruments at the depth of MATS (Fig. 16).

\section{Summary}

Measurements of dissipation rate of TKE from two different instruments have been qualitatively compared to results from the GOTM system modified to account for wave forcing including the injection of TKE into the water column from wave breaking and wave-turbulence interaction. The measurements of dissipation rate were made as profiles using a tethered free-fall microstructure profiler (MSS) deployed from the ship, and as continuous time series at a fixed depth using a moored system (MATS). The dissipation rates observed by shear probes on the MATS were consistent with those obtained by the MSS profiler at the depth of the MATS turbulence sensors, and they showed an enhancement of dissipation rate near the sea surface relative to that predicted by law of the wall. Furthermore, the observations of $\varepsilon$ were compared with the empirical relation of Terray et al. (1996) at the depth of MATS. Both measurements of $\varepsilon$ were fairly consistent with the TA96 estimates of dissipation rates. When normalized by wave parameters following TA96, our $\varepsilon$ measurements compare well with those published by Gerbi et al. (2009), especially at the depth of MATS.

We modified GOTM by including wave breaking effects and the interaction of the mean current shear with the Stokes drift. To estimate wave-current parameterizations, we used data from the high-resolution pressure sensor and the accelerometer sensor mounted on the MATS. Observed enhanced dissipation rates in low wind speeds and TKE injection deep into the water column away from the wave breaking affected zone (a few meters below the sea surface) suggest that wave-turbulence interaction plays a significant role in the regulation of turbulence distribution near the sea surface. To investigate this numerically, we applied two types of parameterizations for wave-turbulence interaction by modifying the $k-\varepsilon$ turbulence closure model. Comparing observations and modeled simulations confirmed that the wave-modified GOTM results, especially derived from
Huang and Qiao (2010) parametrization, can approximately predict the distribution of TKE in the upper ocean by significant deviation from the NW results for the moderate and high wind conditions. Under the low wind speed conditions between days 299 to 300 , observations showed energetic turbulence which could not be captured by any of the model runs and the TA96 parametrization. Generally, these discrepancies can be explained by surface cooling (Fig. 2c, d) or current shear instability (Fig. 11). Calculations of gradient Richardson number suggest that the current shear instability was not able to enhance turbulent mixing and dissipation during the experiment. During periods with energetic wind and waves, near-surface enhancement of TKE and the vertical extent of increased dissipation rates were consistent with the measurements. The agreement with the observations improved when wave forcing, especially Stokes production, is included. Further discrepancies between observed and modeled $\varepsilon$ in some periods of the experiment can partly be attributed to uncertainties in the estimation of wave energy spectrum from a moving platform, spatial separations between measuring systems, in using a nondirectional wave energy spectrum, in wave forcing parameterizations, atmospheric surface forcing, and due to the intermittent characteristic of turbulence microstructure in the water column.

Acknowledgments This work has been partially funded by the Norwegian Center for Offshore Wind Energy (NORCOWE) under grant 193821/S60 from Research Council of Norway (RCN). NORCOWE is a consortium with partners from industry and science, hosted by Christian Michelsen Research. Furthermore, the comments of the anonymous reviewers helped to improve the manuscripts.

\section{References}

Agrawal YC, Terray EA, Donelan MA, Hwang PA, Williams-III AJ, Drennan WM, Kahma KK, Kitaigorodskii SA (1992) Enhanced dissipation of kinetic energy beneath surface waves. Nature 359:219-220

Anis A, Moum JN (1995) Surface wave-turbulence interactions. scaling $\epsilon(z)$ near the sea surface. J Phys Oceanogr 25:20252045

Babanin AV, Haus BK (2009) On the existence of water turbulence induced by nonbreaking surface waves. J Phys Oceanogr 39:26752679

Baker MA, Gibson CH (1987) Sampling turbulence in the stratified ocean: statistical consequences of strong intermittency. J Phys Oceanogr 17:1817-1836

Bakhoday-Paskyabi M, Fer I (2013) Turbulence measurements in shallow water from a subsurface moored moving platform. Energ Proced 35:307-316

Bakhoday-Paskyabi M, Fer I, Jenkins AD (2012) Surface gravity wave effects on the upper ocean boundary layer: Modification of a one-dimensional vertical mixing model. Cont Shelf Res 38:6378

Burchard H (2001) Simulating the wave-enhanced layer under breaking surface waves with two-equation turbulence models. J Phys Oceanogr 31:3133-3145 
Burchard H, Bolding K (2001) Comparative analysis of four second moment turbulence closure models for the oceanic mixed layer. $\mathrm{J}$ Phys Oceanogr 31:1943-1968

Burchard H, Bolding K, Villarreal MR (1999) GOTM, a general ocean turbulence model. Theory, implementation and test cases. Tech Rep EUR 18745, Euro Comm

Bye J (1988) The coupling of wave drift and wind velocity profiles. J Mar Res 46:457-472

Carniel S, Warner J, Chiggiato J, Sclavo M (2009) Investigating the impact of surface wave breaking on modeling the trajectories of drifters in the northern Adriatic Sea during a wind-storm event. Ocean Model 30:225-239

Churchill J, Csanady G (1983) Near-surface measurements of quasiLagrangian velocities in open water. J Phys Oceanogr 13:16691680

Clark N, Eber L, Laurs R, Renner J, Saur J (1974) Heat exchange between ocean and atmosphere in the eastern north Pacific for 1961-71. NOAA Tech Rep NMFS SSRF-682. US Dept of Commer:108

Craig PD (1996) Velocity profiles and surface roughness under breaking waves. J Geophys Res 101(C1):1265-1277

Craig PD, Banner ML (1994) Modeling wave-enhanced turbulence in the ocean surface layer. J Phys Oceanogr 24:2546-2559

Drennan WM, Donelan MA, Terray EA, Katsaros KB (1996) Oceanic turbulence dissipation measurements in SWADE. J Phys Oceanogr $26: 808-815$

Fer I (2006) Scaling turbulent dissipation in an Arctic fjord. Deep Sea Res 53:77-95

Fer I, Bakhoday-Paskyabi M (2014) Autonomous ocean turbulence measurements with a moored instrument. J Atmos Ocean Tech 31: 474-490

Gargett AE (1989) Ocean turbulence. Annu Rev Fluid Mech 21:419_ 451

Gemmrich JR, Farmer DM (1999) Near-surface turbulence and thermal structure in a wind-driven sea. J Phys Oceanogr 29:480499

Gemmrich JR, Farmer DM (2004) Near-surface turbulence in the presence of breaking waves. J Phys Oceanogr 34:1068-1086

Gerbi GP, Trowbridge JH, Terray EA, Plueddemann AJ, Kukulka T (2009) Observations of turbulence in the ocean surface boundary layer: energetics and transport. J Phys Oceanogr 39:10771096

Goodman L, Levine ER, Lueck RG (2006) On measuring the terms of the turbulent kinetic energy budget from an AUV. J Atmos Oceanic Technol 23:977-990

Harcourt RR (2013) A second moment closure model of langmuir turbulence. J Phys Oceanogr 43:in press

Hasselmann K (1970) Wave-driven inertial oscillations. Geophys Fluid Dyn 1:463-502

Huang CJ, Qiao F (2010) Wave-turbulence interaction and its induced mixing in the upper ocean. J of Geophy Res 115. doi: 10.1029/2009JC005,853.

Janssen PAEM (1989) Wave-induced stress and the drag of air flow over sea waves. J Phys Oceanogr 19:745-753

Janssen PAEM (1991) Quasi-linear theory of wind-wave generation applied to wave forecasting. J Phys Oceanogr 21:16311642

Janssen PAEM (2012) Ocean wave effects on the daily cycle in SST. J Geophy Res 117:1-24

Jenkins AD (1987) Wind and wave-induced currents in a rotating sea with depth-varying eddy viscosity. J Phys Oceanogr 17:938951

Jenkins AD (1992) A quasi-linear eddy-viscosity model for the flux of energy and momentum to wind waves, using conservation-law equations in a curvilinear coordinate system. J Phys Oceanogr 22(8):843-858
Jones NL, Monismith SG (2007) The influence of whitecapping waves on the vertical structure of turbulence in a shallow estuarine embayment. J Phys Oceanogr 38:1563-1580

Kantha LH, Clayson CA (2004) On the effect of surface gravity waves on mixing in the oceanic mixed layer. Ocean Modelling 6(2):101124

Kitaigorodskii SA, Donelan MA, Lumley JL, Terray EA (1983) Wave-turbulence interactions in the upper ocean. Part II: statistical characteristics of wave and turbulent components of the random velocity field in the marine surface layer. J Phys Oceanogr 13:1988-1999

Komen G (1987) Energy and momentum fluxes through the sea surface. Dynamics of the ocean surface mixed layer, edited by $\mathrm{P}$ Müller and D Henderson, Hawaii Inst of Geophys, Honolulu pp $207-217$

Kondo J (1975) Air-sea bulk transfer coefficients in diabatic conditions. Bound Layer Meteor 9:91-112

McWilliams JC, Sullivan PP, Moeng CH (1997) Langmuir turbulence in the ocean. J Fluid Mech 334:1-30

Mellor GL, Blumberg AF (2004) Wave breaking and ocean surface layer thermal response. J Phys Oceanogr 34:693-698

Mellor GL, Yamada T (1982) Development of a turbulence closure model for geophysical fluid problems. Rev Geophys Space Phys 20:851-875

Miles JW (1957) On the generation of surface waves by shear flows. J Fluid Mech 3:185-204

Oakey NS (1982) Determination of the rate of dissipation of turbulent energy from simultaneous temperature and velocity shear microstructure measurements. J Phys Oceanogr 12:256-271

Osborn TR, Farmer DM, Vagle S, Thorpe S, Cure M (1992) Measurements of bubble plumes and turbulence from a submarine. Atmos Ocean 30:419-440

Polton JE, Lewis DM, Belcher SE (2005) The role of wave-induced Coriolis-Stokes forcing on the wind-driven mixed layer. J Phys Oceanogr 35:444-457

Qiao F, Yuan Y, Yang Y, Zheng Q, Xia C, Ma J (2004) Wave-induced mixing in the upper ocean: distribution and application to a global ocean circulation model. Jeophys Res Lett:31

Rascle N, Ardhuin F (2009) Drift and mixing under the ocean surface revisited. stratified conditions and model-data comparisons. J Geophys Res: 114

Rascle N, Ardhuin F, Terray EA (2006) Drift and mixing under the ocean surface: a coherent one-dimensional description with application to unstratified conditions. J Geophys Res 111(C3):C03016

Skyllingstad ED, Denbo DW (1995) An ocean large-eddy simulation of Langmuir circulations and convection in the surface mixed layer. J Geophys Res 100:8501-8522

Soloviev A, Lukas R (2003) Observation of wave-enhanced turbulence in the near-surface layer of the ocean during TOGA-COARE. Deep Sea Res I(50):371-395

Stacey MW (1999) Simulation of the wind-forced near-surface circulation in Knight inlet: a parameterization of the roughness length. J Phys Oceanogr 29:1363-1367

Stips A, Burchard H, Bolding K, Prandke H, Simon A, Wüest A (2005) Measurement and simulation of viscous dissipation in the wave affected surface layer. Deep Sea Res II(52):1133-1155

Sullivan PP, McWilliams JC (2010) Dynamics of winds and currents coupled to surface waves. Annu Rev Fluid Mech 42:19-42

Sullivan PP, McWilliams JC, Melville WK (2007) Surface gravity wave effects in the oceanic boundary layer: large-eddy simulation with vortex force and stochastic breakers. J Fluid Mech 593:405452

Tang CL, Perrie W, Jenkins AD, DecTracy BM, Toulany B (2007) Observation and modeling of surface currents on the grand banks: a study of the wave effects on surface currents. J Geophys Res 112:1-16 
Terray EA, Donelan MA, Agrawal YC, Drennan WM, Kahma KK, Williams AJ, Hwang PA, Kitaigorodski SA (1996) Estimates of kinetic energy dissipation under breaking waves. J Phys Oceanogr 26:792-807

Thorpe SA (1995) Dynamical processes of transfer at the sea surface. Prog in Oceanogr 35:315-352

Thorpe SA, Humphries P (1980) Bubbles and breaking waves. Nature 283:463-465

Thorpe SA, Osborn TR, Jackson JFE, Hall AJ, Lueck RG (2003) Measurements of turbulence in the upper-ocean mixing layer using AUTOSUB. J Phys Oceanogr 33:122-145

Umlauf L, Burchard H (2003) A generic length-scale equation for geophysical turbulence models. J of Marin Res 61:235-265
Umlauf L, Burchard H, Hutter K (2003) Extending the $k-\omega$ turbulence model towards oceanic applications. Ocean Modell 5:195218

Veron F, Melville WK (2001) Experiments on the stability and transition of wind-driven water surfaces. J Fluid Mech 446:25-65

Veron F, Melville W, Lenain L (2008) Infrared techniques for measuring ocean surface processes. J Atmos Ocean Tech 25:307326

Wang W, Huang RX (2004) Wind energy input to the surface waves. J Phys Oceanogr 34:1276-1280

Wüest A, Piepke G, Van-Senden D (2000) Turbulent kinetic energy balance as a tool for estimating vertical diffusivity in wind-forced stratified waters. Limnol Oceanogr 45:1388-1400 